Running head: The interoceptive attention scale

Word count: 8303 words

Submission date: $5^{\text {th }}$ November 2020

\title{
Dissociations between interoceptive accuracy and attention: evidence from the interoceptive attention scale
}

\author{
Eleonora Gabriele ${ }^{1 \wedge}$, Ria Spooner $^{2 \wedge}$, Rebecca Brewer $^{2} \&$ Jennifer Murphy*2 \\ ${ }^{1}$ Department of Psychology, Institute of Psychiatry, Psychology and Neuroscience, King's \\ College London \\ ${ }^{2}$ Department of Psychology, Royal Holloway, University of London
}

${ }^{\wedge}$ These authors contributed equally to the paper.

*Corresponding author

Department of Psychology

Royal Holloway, University of London

Egham, Surrey

TW20 0EX

Jennifer.murphy@rhul.ac.uk 


\begin{abstract}
Previous evidence suggests a dissociation between self-reported interoceptive accuracy and self-reported interoceptive attention. However, it remains unclear whether such dissociations are driven by differences in the interoceptive signals rated across these questionnaires, or a genuine dissociation between different facets of interoception (accuracy and attention). Across three studies, we examined the relationship between existing measures of selfreported interoceptive accuracy and self-reported interoceptive attention by developing a novel measure of self-reported interoceptive attention - the Interoceptive Attention Scale (IATS) - designed to match the interoceptive sensations included in an existing questionnaire measure of interoceptive accuracy. In addition, we examined whether the interpretation of questionnaire measures of interoception altered associations across measures. Results support the proposed distinction between self-reported interoceptive attention and self-reported interoceptive accuracy and highlight the importance of considering the interpretation of questionnaire measures of interoception.
\end{abstract}

Key words: Interoception, Interoceptive accuracy, Interoceptive attention, Individual differences 


\section{Highlights}

- Examines relationships between self-reported interoceptive attention and accuracy.

- Results support dissociations between interoceptive attention and accuracy.

- Individuals' interpretations of the BPQ interoception scale varied substantially.

- Interpretation of the BPQ altered associations with IAS and IATS.

- Results highlight a need for scrutiny of self-report measures of interoception. 


\section{Introduction}

In recent years there has been a renewed focus on interoception, the perception of one's own internal bodily states (Khalsa et al., 2018), and its relevance for both higher order cognitive processes and psychopathology. Whilst there is a long theoretical tradition linking interoception to fundamental processes such as emotion, learning, and decision making (Bechara \& Damasio, 2005; Damasio, 1994), contemporary research has begun to test these theoretical predictions (Critchley \& Harrison, 2013). Research of this type suggests that greater interoceptive abilities are associated with enhanced emotion recognition and regulation (Terasawa, Fukushima, \& Umeda, 2013; Zamariola, Frost, Van Oost, Corneille, \& Luminet, 2019), memory for emotional information (Garfinkel et al., 2013; Pollatos \& Schandry, 2008; Werner, Peres, Duschek, \& Schandry, 2010) and decision-making performance (Dunn et al., 2010; Sugawara, Terasawa, Katsunuma, \& Sekiguchi, 2020). Parallel to this renewed interest in the relationship between interoception and cognition, individual differences in interoceptive abilities have increasingly been linked to mental health (see Khalsa et al., 2018; Murphy, Brewer, Catmur, \& Bird, 2017). Indeed, a body of literature suggests the presence of atypical interoceptive abilities across a number of mental health conditions, including anxiety, depression, obsessive compulsive disorder, anorexia nervosa, schizophrenia, borderline personality disorder and substance use disorders (Ardizzi et al., 2016; Eggart, Lange, Binser, Queri, \& Müller-Oerlinghausen, 2019; Löffler, Foell, \& Bekrater-Bodmann, 2018; Paulus \& Stein, 2010; Pollatos et al., 2008; Schultchen, Zaudig, Krauseneck, Berberich, \& Pollatos, 2019; Sönmez, Kahyacı Kılıç, Ateş Çöl, Görgülü, \& Köse Çınar, 2017). Despite these considerable efforts to develop our understanding of interoception, and determine its relevance for health and cognition, empirical progress has been impeded by difficulties with the measurement of interoceptive abilities. 
It has been proposed that individual differences in interoception should be considered within a three-dimensional classification framework (Garfinkel, Seth, Barrett, Suzuki, \& Critchley, 2015). This framework distinguishes between (1) interoceptive accuracy, namely how objectively accurate an individual is at detecting internal signals (assessed using objective measures such as heartbeat counting or detection tasks; for an overview see Brener \& Ring, 2016); (2) interoceptive sensibility, referring to an individual's belief in their interoceptive ability and the degree to which they feel engaged by interoceptive signals (assessed by selfreport measures such as confidence ratings collected during a task of interoceptive accuracy or questionnaires e.g. the Body Perception Questionnaire (BPQ); Porges, 1993); (3) interoceptive awareness, a metacognitive measure reflecting the correspondence between objectively measured interoceptive accuracy and self-reported interoceptive sensibility. This framework importantly highlights a need to separate subjective and objective facets of interoception, emphasises the importance of interoceptive awareness, and has been used in numerous investigations (e.g., Forkmann et al., 2016; Garfinkel, Tiley, et al., 2016; Palser, Fotopoulou, Pellicano, \& Kilner, 2018). Recent data suggest, however, that there may be additional facets that need to be incorporated within this framework. For example, although this model classifies self-report measures under the umbrella of interoceptive sensibility, differential relationships have been reported to exist within the interoceptive sensibility dimension (e.g., questionnaire measures and confidence ratings do not usually correlate with each other; Garfinkel et al., 2015). Likewise, whilst this model highlights separation of interoceptive sensibility and objective interoceptive accuracy, research suggests that subjective confidence ratings sometimes correlate with objective interoceptive accuracy, whilst questionnaire measures usually do not (Ferentzi, Drew, Tihanyi, \& Köteles, 2018; Garfinkel et al., 2015; Murphy, Brewer, et al., 2020; though this may depend on the 
interoceptive accuracy task utilised; e.g., Forkmann et al., 2016; Schulz, Lass-Hennemann, Sütterlin, Schächinger, \& Vögele, 2013).

Murphy, Catmur, and Bird, (2019) proposed that the aforementioned discrepancies may be understood by considering not only how interoception is measured (e.g., the level of measurement: objective vs. subjective) but also what is measured (Figure 1). Whilst their $2 \mathrm{x}$ 2 factorial model of interoception, like the tripartite model, highlights a need to separate measures depending on whether the measure provides an objective estimate or is based on self-report, this model also highlights a need to separate measures according to whether they assess interoceptive accuracy or attention. As such, the $2 \times 2$ model promotes four core facets of interoceptive ability: (1) objectively measured interoceptive accuracy, (2) self-reported interoceptive accuracy, (3) objectively measured interoceptive attention and (4) self-reported interoceptive attention, and suggests that interoceptive awareness may be assessed separately for interoceptive attention and accuracy, by examining both the correspondence between selfreported accuracy and objective accuracy, and that between self-reported attention and objective attention (see Figure 1). Importantly, previously reported discrepancies between facets of interoception may be understood within this framework; the lack of a relationship between confidence ratings (a measure of self-reported interoceptive accuracy) and questionnaires such as the BPQ (a measure of self-reported interoceptive attention; Garfinkel et al., 2015) may be due to the fact that these measures assess different aspects of interoception (accuracy and attention, respectively). Likewise, objective measures of interoceptive accuracy may correlate with confidence ratings (a measure of self-reported accuracy) but not questionnaires such as the BPQ (a measure of self-reported attention) because they assess similar and dissimilar aspects of interoception, respectively, despite differences in the level of measurement (Garfinkel et al., 2015; Murphy et al., 2019). Direct support for this model comes from recent research assessing the relationship between 
questionnaire measures of interoceptive ability (e.g., Murphy, Brewer, et al., 2020). Across a number of studies, it was found that whilst measures of self-reported accuracy were generally correlated with one another (e.g. confidence ratings collected during an objective interoceptive accuracy task, the Interoceptive Accuracy Scale (IAS); Murphy, Brewer, et al., 2020), and the Interoceptive Confusion Questionnaire (ICQ); Brewer, Cook, \& Bird, 2016) these measures were uncorrelated with a measure of self-reported interoceptive attention (the BPQ; Porges, 1993). Furthermore, relationships between objective interoceptive accuracy and self-reported interoception were only found when self-report measures assessed interoceptive accuracy and not interoceptive attention.

Despite evidence in support of this 2 x 2 model of interoception, certain differences across these questionnaires prompt an alternative explanation. Indeed, whilst the lack of association between the questionnaire measures of self-reported accuracy (IAS and ICQ) and attention (BPQ) may be due to differences in the facet of interoception assessed (accuracy and attention, respectively), it is also possible that dissociations are due to differences in the interoceptive sensations rated across each questionnaire. Such an alternative explanation is plausible given evidence that objectively measured interoceptive accuracy may dissociate across domains (e.g., Ferentzi, Bogdány, et al., 2018; Garfinkel, Manassei, et al., 2016; Steptoe \& Vögele, 1992; but see Herbert, Muth, Pollatos, \& Herbert, 2012; Whitehead \& Drescher, 1980). As such, the aim of the present series of studies was to develop a novel selfreport measure of interoceptive attention - the Interoceptive Attention Scale (IATS) - and assess the relationship between this measure and existing measures of self-reported interoceptive accuracy (IAS and ICQ) and attention (BPQ). Crucially, in order to establish whether the lack of relationship reported between the IAS/ICQ and BPQ was due to differences in the internal signals rated on these questionnaires, rather than the facet of interoception assessed, the IATS was designed such that the signals were matched to the IAS. 
Given this design, observing a relationship between the newly developed IATS and IAS, but not between the BPQ and IAS, would indicate that previous reports of a lack of a relationship between self-reported interoceptive accuracy and attention were driven by differences in the sensations examined by each questionnaire. Conversely, a lack of relationship between the IATS and IAS would indicate a genuine dissociation between self-reported interoceptive attention and accuracy, providing crucial support for the $2 \times 2$ model of interoceptive abilities. In Study 1, we examined the relationship between the IAS, ICQ, IATS and BPQ. Notably, we have previously proposed that the BPQ be used as a measure of self-reported attention, rather than accuracy, as this questionnaire utilises the term 'awareness', and one must attend to one's internal signals in order to become and remain aware of them. As terminology explicitly relating to attention is not used in the BPQ, however, it is possible that individual differences exist in participants' interpretation of this questionnaire, and that these differences affect relationships between measures. In Study 2, therefore, we examined whether participants vary in their interpretation of the BPQ, and whether this interpretation alters associations between the BPQ and measures of self-reported interoception that explicitly specify the interoceptive domain of interest, namely accuracy (IAS) and attention (IATS). Finally, in Study 3, we examined the test-retest reliability of the IATS.

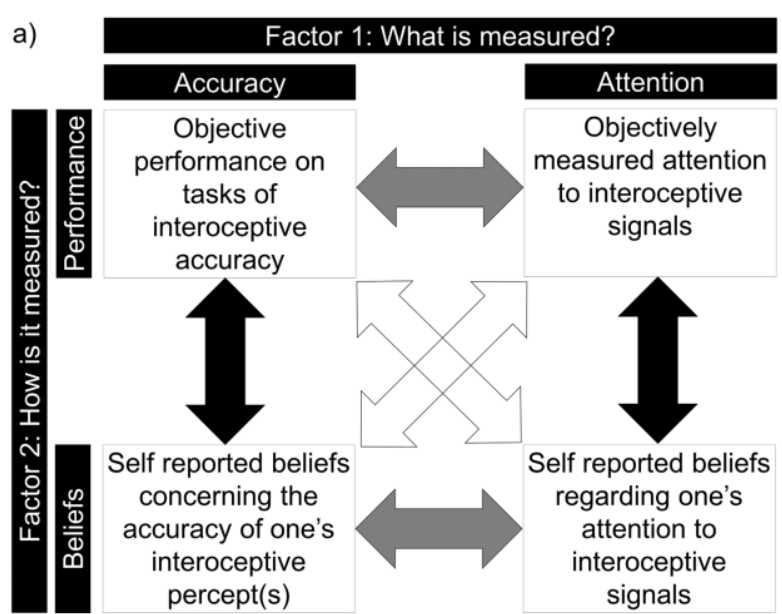

b)

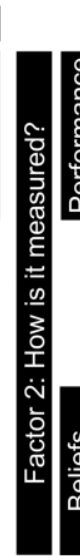

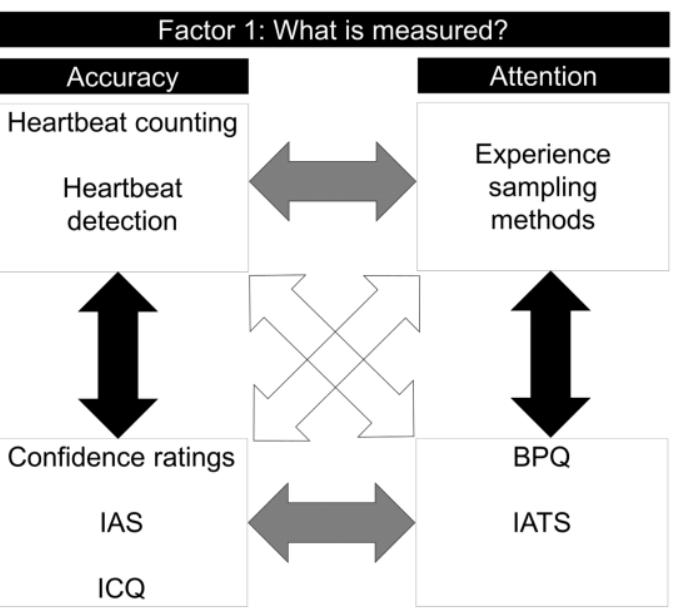


Figure 1. Depiction of the $2 \times 2$ factorial structure of interoceptive abilities proposed by (Murphy et al., 2019). Factor 1 distinguishes between measures of interoceptive accuracy and interoceptive attention. Factor 2 distinguishes between measures of objective performance and self-report measures of beliefs. Four facets are thus defined: (1) objective interoceptive accuracy; (2) self-reported beliefs concerning one's interoceptive accuracy; (3) objective interoceptive attention; and (4) self-reported beliefs concerning one's interoceptive attention. For both accuracy and attention, interoceptive awareness (metacognition) can be quantified by comparing one's self-reported beliefs to the objective measure (black arrows). Correspondence across measures within the same measurement factor can be quantified (grey arrows) as well as the relationship across different measurement and performance factors (white arrows). (b) Illustrative tasks that may index distinct facets of the model. Interoceptive Accuracy Scale (IAS; Murphy, Brewer, et al., 2020; e.g. items such as "I can always accurately perceive when my heart is beating fast"). Interoceptive Confusion Questionnaire (ICQ; e.g., items such as "I am very sensitive to changes in my heart-rate"; Brewer et al., 2016). Porges Body Perception Questionnaire (BPQ; items such as "during most situations I am aware of how hard my heart is beating"; Porges, 1993); Interoceptive Attention Scale (IATS; e.g. items such as 'Most of the time my attention is focused on whether my heart is beating fast'; developed in the current study).

\section{The Interoceptive Attention Scale (IATS)}

The IATS was constructed to quantify the extent to which internal signals are the object of one's attention. As the aim of this project was to assess whether a relationship between selfreport measures of interoceptive attention and accuracy can be found when measures assess the same internal signals, we constructed the IATS ensuring that (where possible) the signals included in the IATS exactly matched the signals included in the IAS. If this resulted in unusual wording (e.g., for affective touch or for bruising) the items were reworded for clarity, but focus was retained on the same internal signal (see Figure 2). Given that attention to internal signals may be a product of both 1) monitoring of the signal (e.g., checking whether one needs to urinate) and 2) the presence of the signal (e.g., the objective activation of stretch 
receptors in the bladder when full, inducing the urge to urinate), the instructions for the IATS explicitly outlined that individuals should rate the extent to which attentional focus is on these signals regardless of whether the signal is present. The wording of individual items was also used to emphasize this (e.g., whether I need to urinate rather than e.g. 'when' or 'that' I need to) and for further clarity specific examples of what would, and would not, constitute attention to internal signals were given in the instructions (see Figure 2). Like the IAS, the IATS scale comprises 21 -items rated from strongly agree (5) to strongly disagree (1), with scores ranging from 21 to 105 . Higher scores indicate greater self-reported attention to internal signals. 
Below are several statements regarding how much attention you pay to specific bodily sensations. Please rate on the scale how much attention you think you pay to each specific sensation. Think about how you feel during most situations in your daily life, rather than at a specific point in time. For example, if you often think about your heart beating, feeling hungry or needing the toilet then you would rate your attention to these sensations as high. In contrast, if you don't often think about your heart rate, how hungry you are or whether you need the toilet then you would rate your attention to these sensations as low.

Please only rate how much attention you pay to these sensations regardless of how well you think you can perceive them. For example, if you often feel you need the toilet but when you go to the toilet you realise you don't need to you should still rate your attention to this signal as high. Do not worry about how often you think the sensation is truly happening inside your body - we would like to know how much of the time you pay attention to these sensations.

The questions ask about your attention to feelings coming from inside your body. For example, if the question asks about temperature, it is referring to sensations you notice internally without using your hand to feel how warm your skin is, and if it asks about your heartbeat, it is referring to feelings you notice inside your body without taking your pulse.

1. Most of the time my attention is focused on whether my heart is beating fast

2. Most of the time my attention is focused on whether I am hungry

3. Most of the time my attention is focused on whether I am breathing fast

4. Most of the time my attention is focused on whether I am thirsty or dehydrated

5. Most of the time my attention is focused on whether I need to urinate

6. Most of the time my attention is focused on whether I need to defecate

7. Most of the time when I am eating, my attention is focused on different tastes

8. Most of the time my attention is focused on whether I am nauseated or need to vomit

9. Most of the time my attention is focused on whether I need to sneeze

10. Most of the time my attention is focused on whether I need to cough

11. Most of the time my attention is focused on the temperature of my body (feeling hot or cold)

12. Most of the time my attention is focused on whether I am sexually aroused

13. Most of the time my attention is focused on whether I need to pass wind

14. Most of the time my attention is focused on whether I need to burp

15. Most of the time my attention is focused on whether my muscles are tired or sore

16. Most of the time my attention is focused on whether I am in pain after I am hurt or injured

17. Most of the time my attention is focused on whether I am in pain (that is not caused by injury)

18. Most of the time my attention is focused on whether my blood sugar is low

19. Most of the time when someone is touching me, my attention is focused on whether it is pleasant/affectionate

20. Most of the time my attention is focused on whether touch or materials feel ticklish on my body

21. Most of the time my attention is focused on whether my body feels itchy

Scale: Strongly Agree (5), Agree (4), Neither agree nor disagree (3), Disagree (2), Disagree Strongly (1).

Figure 2. Interoceptive Attention Scale (IATS). 


\subsection{Ethical approval, pre-registration, and data availability}

For all studies, ethical approval was granted by the local ethics committee following their reviewing procedures. Participants provided informed consent and were fully debriefed upon study completion. Data and pre-registrations of predictions for all studies can be found at [https://osf.io/3v9ea/].

\section{Study 1: Psychometric properties of the IATS and its relationship with existing measures}

Study 1 sought to assess the relationship between the IATS and existing self-report measures of accuracy and attention. In addition, the psychometric properties of the IATS were examined.

\subsection{Methods}

Participants were recruited via social-media and pre-existing databases of individuals who had expressed an interest in taking part in psychological research. Participation was incentivised by a prize draw. Participants completed the IATS alongside three existing interoceptive questionnaires, the Interoceptive Accuracy Scale (IAS; Murphy, Brewer, et al., 2020), the Interoceptive Confusion Questionnaire (ICQ; Brewer et al., 2016) and the awareness scale of the Body Perception Questionnaire (BPQ; Porges, 1993) via Qualtrics (Provo, UT). The order of questionnaire completion was randomised across participants. A minimum sample size of 350 was determined a-priori based on the minimum sample size required for factor analysis (Field, 2005). As detailed in the pre-registration, recruitment was set to continue until $31^{\text {st }}$ July 2020 with the sample size achieved by this date used for analyses so long as it exceeded the minimum sample size of 350 participants. After the removal of duplicate completions (assessed on the basis of duplicate email addresses) and incomplete responses, the final sample comprised 596 individuals $\left(M_{\text {age }}=30.97\right.$ years, $\mathrm{SD}_{\text {age }}$ 
$=12.57$ years, age-range $=18-93$ years, 143 male, 443 female, 10 other). Of these

individuals, $25.3 \%$ reported pre-existing psychiatric conditions (2.5\% declined to answer this question), $22.5 \%$ reported pre-existing physical health conditions (1.7\% declined to answer this question) and $26.7 \%$ reported having English as an additional language.

\subsection{Results}

\subsubsection{Planned analyses}

\subsubsection{Psychometric properties of the IATS}

Given that the primary aim of this project was to assess the relationship between the IATS and other self-report measures of interoception, for brevity the psychometric properties of the IATS are reported in the supplementary information [S1-S3]. In summary, good internal consistency (Cronbach's Alpha) was observed for all measures and a three-factor solution was observed for the IATS.

\subsubsection{Correlations between measures}

Prior to assessing the relationship between measures, assumptions of normality were checked using histograms and Kolmogorov-Smirnov tests and the data were checked for outliers. No outliers ( $>3 x$ the interquartile range) were detected. In terms of normality, although visual inspection of the histograms suggested little deviation from normality, Kolmogorov-Smirnov tests were significant for all questionnaire variables (all $p<.05$ ). As such, both Pearson and Spearman correlations are reported below. All reported correlations are two-tailed. Given that the overall aim of this study was to assess the relationship between questionnaire measures of interoceptive attention and accuracy when measures assess the same internal signals, total scores derived from all items were employed for correlational analyses as detailed in the preregistration. 
As predicted, questionnaire measures of interoceptive accuracy (the IAS and ICQ) were correlated with each other (Pearson: $r(594)=-.644, p<.001$; Spearman: $r(594)=-.610, p<.001)$, suggesting that, as self-reported interoceptive accuracy (IAS scores) increased, self-reported interoceptive confusion decreased. Likewise, measures of self-reported interoceptive attention (the BPQ and IATS) were correlated with each other (Pearson: $r(594)=.336$, $p<.001$; Spearman: $r(594)=.354, p<.001)$. In terms of the relationship across measures, the IAS was not significantly correlated with the BPQ (Pearson: $r(594)=.028, p>.250$; Spearman: $r(594)=-.023, p>.250)$ or the IATS (Pearson: $r(594)=-.016, p>.250$; Spearman: $r(594)=-.072, p=.077)$. Contrary to predictions, the ICQ showed a small positive relationship with the BPQ when non-parametric analysis was used (Pearson: $r(594)=.062$, $p=.129$; Spearman: $r(594)=.130, p=.002)$ and the ICQ was correlated with the IATS (Pearson: $r(594)=.137, p=.001$; Spearman: $r(594)=.197, p<.001)$. In both cases, greater selfreported attention to internal signals was associated with increased interoceptive confusion (see Figure 3).

To compare the size of the correlations between measures designed to assess the same facet (e.g., the correlation between the IAS and ICQ; the correlation between the BPQ and IATS) with measures assessing different facets (e.g., IAS to BPQ; IAS to IATS; ICQ to BPQ and ICQ to IATS) R-to-Z tests were employed (Steiger, 1980; conducted using the quantpsy web implementation; Lee \& Preacher, 2013). Given that directional predictions were made, 1tailed tests were utilised as detailed in the pre-registration. For ease of comparison with the IAS, the ICQ scores were reversed prior to comparing correlation sizes, so that a higher score reflects greater, rather than lower, self-reported accuracy.

For Pearson correlations, the size of the correlation between the two accuracy measures (IAS and ICQ) was significantly larger than the size of the correlation between measures assessing different facets of interoception (IAS with BPQ; IAS with IATS; ICQ with BPQ and; ICQ 
with IATS; all $p<.001$ ), in line with predictions. Likewise, the size of the correlation between the two attention measures (BPQ and IATS) was significantly larger than the size of the correlation between measures assessing different facets (IAS with BPQ; IAS with IATS; ICQ with BPQ and; ICQ with IATS; all $p<.001)$. The same pattern of significance was observed when Spearman correlations were compared (for details please see supplementary information [S4]).
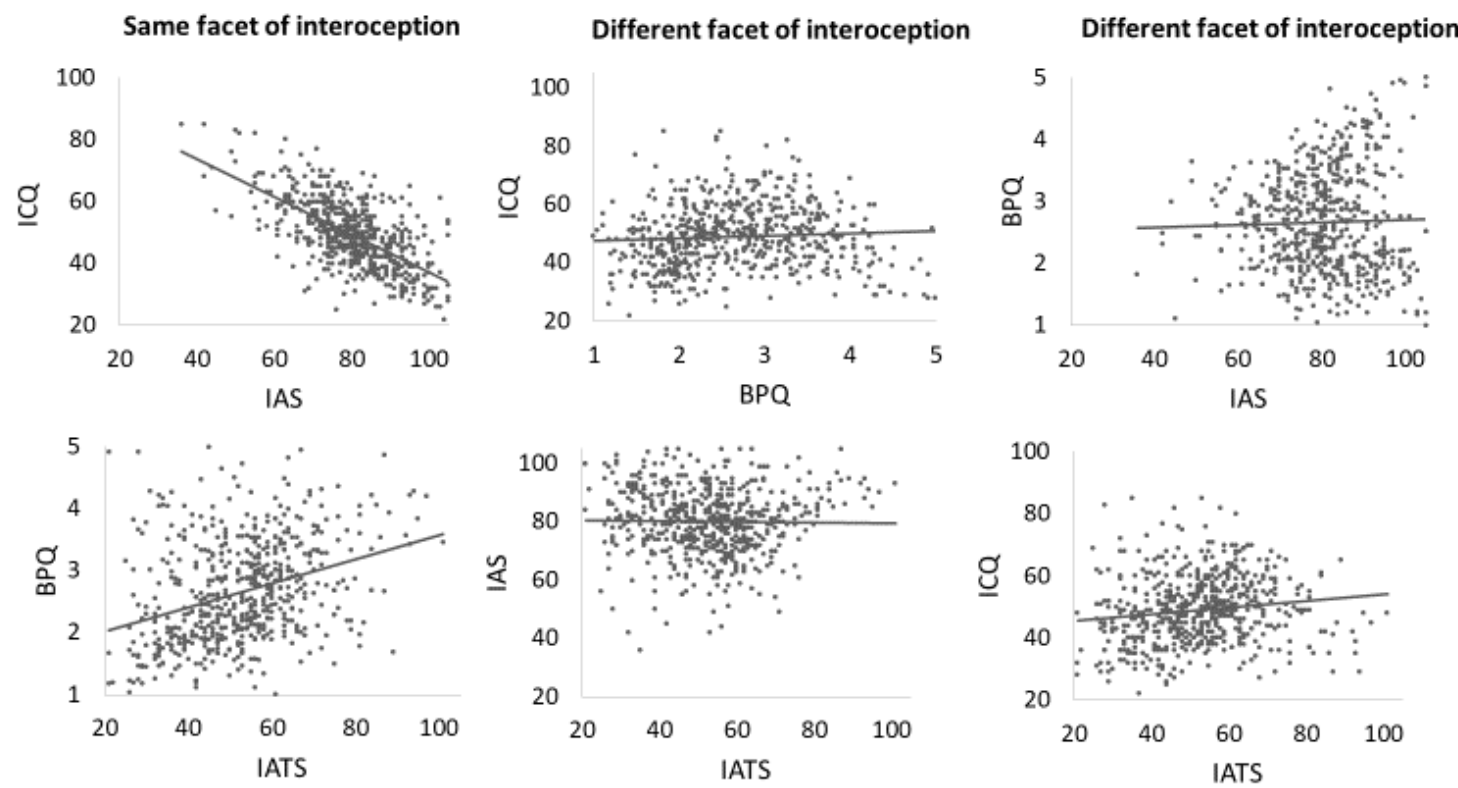

Figure 3. Scatter plots showing correlations from Study 1. IATS = Interoceptive Attention Scale (high scores $=$ greater interoceptive attention). $B P Q=$ Body Perception Questionnaire (high scores $=$ greater interoceptive attention). IAS $=$ Interoceptive accuracy scale (high scores $=$ greater interoceptive accuracy). ICQ = Interoceptive Confusion Questionnaire (high scores $=$ greater interoceptive confusion).

\subsubsection{Planned exploratory analyses}

To examine whether the pattern of results reported above held after the exclusion of individuals reporting mental health conditions and English as an additional language, the 
above analyses were repeated after the removal of these individuals as well as individuals who declined to answer this question. In terms of the correlations between measures, the removal of these individuals had little effect on the relationships reported above (see supplementary information [S5]) except a significant negative correlation between the IATS and IAS was observed, whereby higher self-reported interoceptive attention correlated with lower self-reported interoceptive accuracy (Pearson: $r(303)=-.121, p=.034$; Spearman: $r(303)$ $=-.180, p=.002)$. As in the total sample, however, comparisons of the size of correlations indicated that correlations between measures assessing the same facet of interoception (accuracy or attention) were significantly larger than those between measures assessing different facets (all $p<.001$ ). For details please see supplementary information [S5].

\subsubsection{Unplanned exploratory analyses}

To examine whether the pattern of results reported above held after the exclusion of individuals reporting physical health conditions (and those who declined to specify) in addition to those reporting mental health conditions (and those who declined to specify) and English as an additional language, the above correlational analyses were repeated after the removal of these individuals $(\mathrm{N}=237)$. This had no substantial impact on the pattern of significance reported above, though no association between the ICQ (interoceptive accuracy measure) and BPQ (interceptive attention measure) was observed in this subsample.

Consistent with the other samples, correlations between measures assessing the same facet were greater than measures assessing different facets of self-reported interoception (for details please see supplementary information [S6]).

To compare the size of the correlations between measures of self-reported accuracy (IAS and ICQ) and self-reported interoceptive attention (IATS and BPQ) in the total sample, an R-to-Z test was conducted (Steiger, 1980) implemented via the quantpsy web implementation (Lee 
\& Preacher, 2013). This analysis indicated that the size of the correlation between the IAS and ICQ was significantly greater than the size of the correlation between the IATS and BPQ (comparison of Pearson correlations: $Z=7.11, p<.001$; comparison of Spearman correlations: $Z=5.82, p<.001$ ). This pattern of results was also consistent in the first (comparison of Pearson correlations: $Z=3.63, p<.001$; comparison of Spearman correlations: $Z=3.44$, $p<.001$ ) and second (comparison of Pearson correlations: $Z=2.41, p<.01$; comparison of Spearman correlations: $Z=2.46, p<.01)$ sub-samples.

Given recent suggestions (Campos et al., in prep) that there may be a quadratic association between self-reported attention and self-reported accuracy, we also explored the possibility of non-linear associations between these variables. As noted in Campos et al., (in prep), in all models accuracy was treated as the dependent variable and attention as the independent variable. We found some evidence for a small quadratic association between accuracy and attention measures, whereby both lower and higher levels of self-reported interoceptive attention were associated with greater self-reported interoceptive accuracy than mid-levels of interoceptive attention. However, some evidence of cubic associations was also observed (see supplementary information [S7]).

\subsection{Discussion}

In Study 1, a consistent pattern emerged across all planned and exploratory analyses whereby the IAS (a measure of self-reported interoceptive accuracy) was correlated with the ICQ (a measure of accuracy) but not the BPQ (a measure of attention). Likewise, a consistent positive relationship was observed between the BPQ and the IATS (both measures of interoceptive attention). In terms of the relationships across measures thought to be assessing different facets, where relationships were observed these were small and in an unexpected direction, with greater attention to internal signals relating to lower accuracy. Comparisons of 
the size of these correlations consistently demonstrated stronger relationships between measures assessing the same facet of interoception (accuracy (e.g., IAS and ICQ) or attention (e.g., IATS and BPQ)) when compared to the relationship between measures assessing different facets.

It is notable, however, that unplanned exploratory analyses demonstrated a stronger relationship between the measures of interoceptive accuracy (ICQ and IAS) than between the measures of interoceptive attention (BPQ and IATS). In interpreting this finding, it is of relevance that the BPQ terminology requires participants to report their level of 'awareness' of internal bodily signals rather than explicitly assessing the extent to which internal signals are the object of their attention. It is possible, therefore, that individual differences in the interpretation of the term 'awareness' (where some participants interpret the term awareness as referring to attention and others interpret the term as referring to accuracy, the extent to which these signals occur in one's body, or otherwise) may underlie the smaller relationship observed between the IATS and BPQ. This possibility was examined in Study Two.

\section{Study 2 (interpretation of the questionnaires)}

The aim of Study 2 was to further assess the relationship between self-reported attention and accuracy and examine whether participants' interpretations of the questionnaires alters associations. Specifically, given that the term 'awareness' used in the BPQ is ambiguous, and may be interpreted as asking about attention, accuracy, presence of physiological signals, or otherwise, the aim of this study was to investigate how the interpretation of the BPQ might alter associations between the BPQ and measures of attention (the IATS) and accuracy (the IAS).

As in Study 1, predictions were pre-registered prior to data collection (https://osf.io/mkhby). It was predicted that within a sub-sample of individuals who interpret the BPQ as assessing 
accuracy, a positive relationship would be observed between the BPQ and IAS, and no relationship would be observed between the BPQ and IATS. The size of the relationship between the BPQ and IAS was also predicted to be significantly larger than the relationship between the BPQ and IATS in this sub-sample. Conversely, in a sub-sample of individuals who interpret the BPQ as assessing attention, a positive relationship was predicted between the IATS and BPQ, while no relationship was predicted between the BPQ and IAS. The size of the relationship between the BPQ and IATS was also predicted to be significantly larger than the relationship between the BPQ and IAS in this sample. In the total sample, predictions were the same as in Study 1.

\subsection{Methods}

Participants were recruited via social media outlets and pre-existing databases of individuals who had expressed an interest in taking part in psychological research. Entry into a prize draw was offered as an incentive. Participants completed the IATS, IAS and BPQ in a randomised order online via Qualtrics (Provo, UT). At the end of each questionnaire, participants were asked how they interpreted the questionnaire and a free text box was given for further comments (e.g. IATS: 'In this questionnaire we asked you to tell us how much attention you pay to specific bodily sensations. While you were completing the questionnaire, what did you think the term 'attention' meant in this context? A) How much attention you pay to these sensation, B) How accurate you are at perceiving these sensations, C) How often (frequently) or intensely these sensations actually occur in your body'; for details please see supplementary information [S8]). As detailed in the pre-registration, data collection was set to continue until there were either 123 participants in each group (123 who believed the BPQ was assessing accuracy and 123 who believed the BPQ was assessing attention, providing $>80 \%$ power to detect an effect size of $r=.25$ ) or at least 500 participants had completed the survey. Having removed duplicate completions and incomplete responses, the final sample 
was comprised of 500 individuals $\left(\mathrm{M}_{\mathrm{age}}=33.60\right.$ years, $\mathrm{SD}_{\mathrm{age}}=15.16$ years, Age range $=18$ 89 years, 121 Males, 374 Females, 5 Other). Of these individuals, 24\% reported pre-existing psychiatric conditions ( $2 \%$ declined to answer this question), $25.2 \%$ reported pre-existing physical health conditions ( $0.4 \%$ declined to answer this question) and $22.6 \%$ reported English as a second language.

\subsection{Results and discussion}

\subsubsection{Planned analyses}

In the total sample, the majority of participants interpreted the IATS as a measure of attention (79\%), with fewer interpreting the IATS as assessing accuracy (9.6\%), how often the signal objectively occurs $(10.8 \%)$ or otherwise $(0.6 \%$; see Figure 4$)$. Of these three 'other' responses, one individual interpreted the IATS as measuring 'all of the above' with the other two individuals providing no further clarification. For the IAS, the majority interpreted the measure as assessing accuracy $(84.6 \%)$, followed by attention $(10.2 \%)$, how often the signal objectively occurs $(4.6 \%)$ or other $(0.6 \%)$. Of the three individuals answering 'other', one individual declined to provide specification, one interpreted the IAS as assessing 'all of the above' and one interpreted the IAS as assessing how often they remember or notice signals during or after they have occurred. For the BPQ, there was substantially less consensus across responses; the most common interpretation was that the BPQ was assessing attention (36.4\%), followed by how often the signal objectively occurs $(30.8 \%)$, accuracy $(30.4 \%)$ and other $(2.4 \%)$. Content analysis of other responses for the BPQ suggested the individuals interpreted the BPQ as assessing either 'all of the above', a combination of accuracy and attention or how often they are aware of/notice/perceive/are conscious of the sensation regardless of/or dependent on the presence of the signal (for full details of other responses see supplementary information [S9]). 


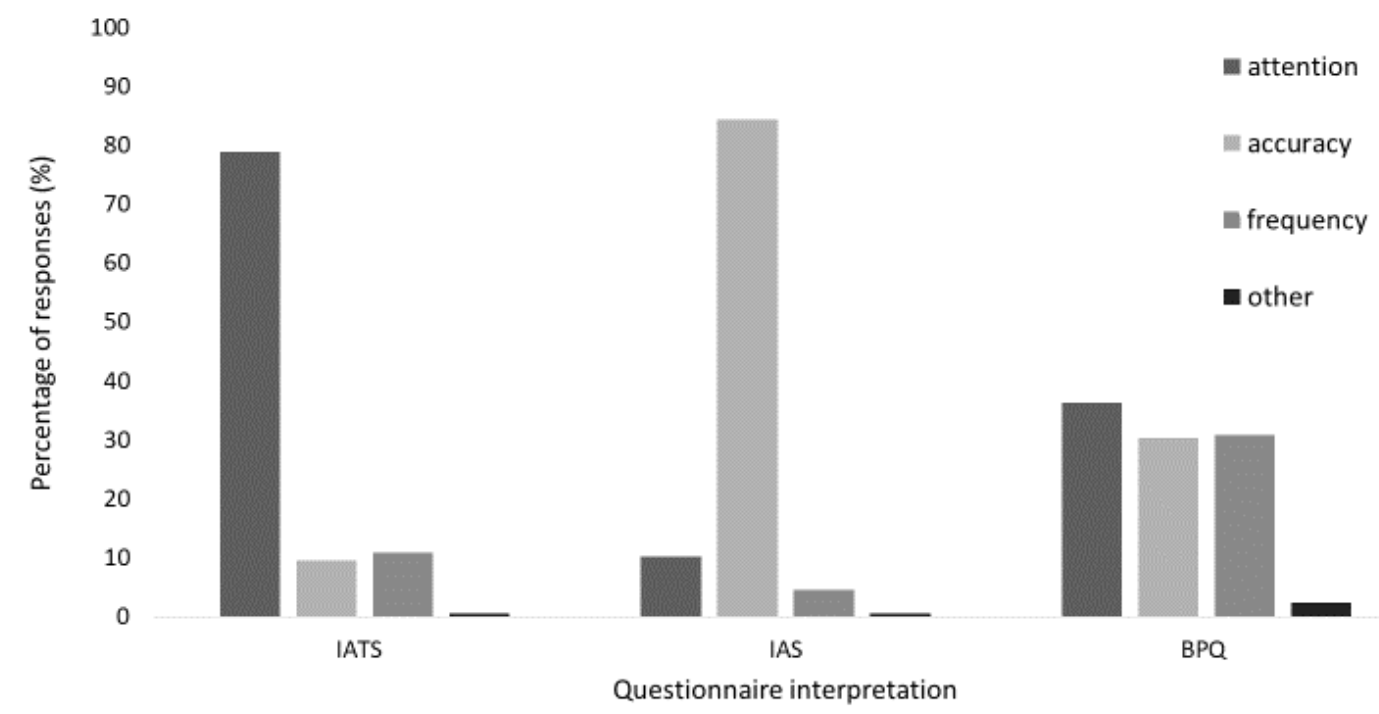

Figure 4. Bar graph depicting the proportion of participants who interpreted each questionnaire as assessing attention, accuracy, how often the signal objectively occurs or other. $I A T S=$ Interoceptive Attention Scale. $B P Q=$ Body Perception Questionnaire. IAS $=$ Interoceptive accuracy scale.

Prior to assessing the relationship between measures, assumptions of normality were checked using histograms and Kolmogorov-Smirnov tests and the data were checked for outliers. No outliers ( $>3 \mathrm{x}$ the interquartile range) were detected. Where normality was concerned, although visual inspection of the histograms suggested little deviation from normality, Kolmogorov-Smirnov tests were significant for all questionnaire variables (all $p<.05$ ) except the IATS $(p=.179)$. As such, both Pearson and Spearman correlations are reported below. All reported correlations are two-tailed.

Correlations were first assessed in the total sample. Consistent with Study 1, a significant relationship between the IATS and BPQ was observed (Pearson: $r(498)=.317, p<.001$; Spearman: $r(498)=.337, p<.001)$. No correlation was observed between the IAS and IATS (Pearson: $r(498)=.058, p=.195$; Spearman: $r(498)=.036, p>.250)$. In contrast to Study 1 , a 
significant relationship was observed between the IAS and BPQ when parametric analyses were used (Pearson: $r(498)=.153, p=.001$; Spearman: $r(498)=.087, p=.053)$.

To examine whether the relationships between the BPQ and the IATS and IAS vary depending on participants' interpretation of the measures, the relationships between the measures were examined as a function of interpretation. As specified in the pre-registration, we excluded individuals who did not interpret the IAS as assessing accuracy, and the IATS as assessing attention, from all interpretation analyses given that these terms are explicitly used in these questionnaires. In the sub-sample who interpreted the BPQ as assessing attention, a significant association was observed between the BPQ and IATS (Pearson: $r(121)=.463$, $p<.001$; Spearman: $r(121)=.459, p<.001)$. No relationship was observed between the BPQ and IAS (Pearson: $r(121)=-.085, p>.250$; Spearman: $r(121)=-.121, p=.181)$ or the IAS and IATS (Pearson: $r(121)=.004, p>.250$; Spearman: $r(121)=.004, p>.250)$ (Figure 5) in this subsample, as expected.

In contrast, in the sub-sample who interpreted the BPQ as assessing accuracy, a significant association was observed between the BPQ and IAS when parametric analyses were used, with a trend observed in the non-parametric analysis (Pearson: $r(95)=.246, p=.015$; Spearman: $r(95)=.187, p=.067)$. No association was observed between the BPQ and IATS (Pearson: $r(95)=.030, p>.250$; Spearman: $r(95)=.090, p>.250)$ or the IATS and IAS (Pearson: $r(95)=-.073, p>.250$; Spearman: $r(95)=-.083, p>.250)$ in this sub-sample, again as predicted. R-to-Z tests were used to compare the size of these correlations (Cohen, \& Cohen, 1983; implemented via the quantpsy web implementation; Preacher, 2002). 1-tailed tests are reported, in line with directional hypotheses. As predicted, the size of the correlation between the BPQ and IATS was significantly larger in the sub-sample who interpreted the BPQ as assessing attention, than the sub-sample who interpreted the BPQ as assessing accuracy 
(Pearson: $Z=3.42, p<.001$; Spearman: $Z=2.95, p=.002$ ). Likewise, the size of the correlation between the BPQ and IAS was significantly larger in the sub-sample who interpreted the BPQ as assessing accuracy, when compared to the sub-sample who interpreted the BPQ as assessing attention (Pearson: $Z=-2.44, p=.007$; Spearman: $Z=-2.26, p=.012$ ).

\section{Sub-sample who interpreted the BPQ as assessing attention}
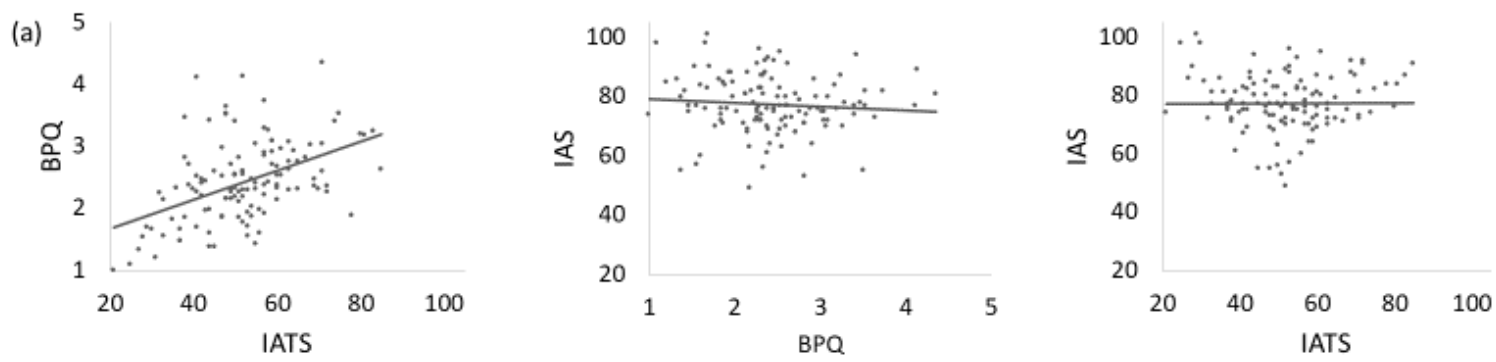

Sub-sample who interpreted the BPQ as assessing accuracy
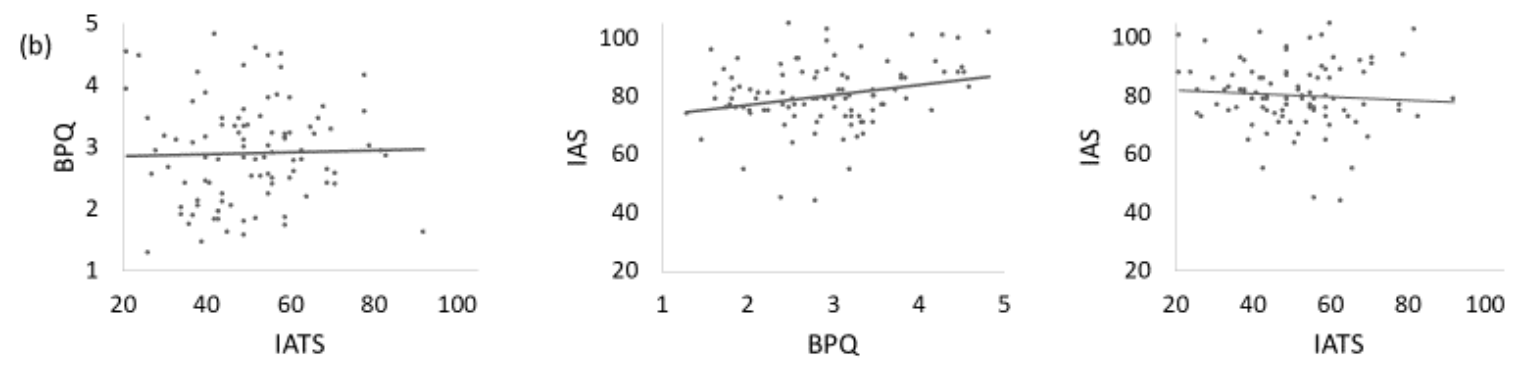

Figure 5. Correlations from Study 2. (a) Depicts the relationship between the three questionnaires within the sub-sample where the BPQ was interpreted as assessing attention. (b) Depicts the relationship between the three questionnaires within the sub-sample where the $B P Q$ was interpreted as assessing accuracy. IATS = Interoceptive Attention Scale. $B P Q$ = Body Perception Questionnaire. IAS = Interoceptive accuracy scale.

\subsubsection{Planned exploratory analyses}

The above analyses were re-run excluding individuals who reported mental health conditions (or declined to specify) or English as an additional language ( $\mathrm{N}=282)$. Exclusion of these individuals did not notably change the pattern of significance in comparison to the total sample correlations (see supplementary information [S10]). In the sub-sample who 
interpreted the BPQ as assessing attention, again an association between the IATS and BPQ was observed (Pearson: $r(65)=.583, p<.001$; Spearman: $r(65)=.561, p<.001$ ). Whilst an association between the IAS and BPQ was observed in this sub-sample for non-parametric analyses, this association was negative, whereby higher attention, assessed by the BPQ, was associated with lower self-reported accuracy, assessed by the IAS (Pearson: $r(65)=-.220$, $p=.074$; Spearman: $r(65)=-.258, p=.035)$. No association was observed between the IATS and IAS (Pearson: $r(65)=-.149, p=.227$; Spearman: $r(65)=-.117, p>.250)$. Conversely, in this sub-sample no association was observed between any of the measures when individuals interpreted the BPQ as assessing accuracy (all $p>.05$ ), though a trend was observed between the BPQ and IAS when parametric analyses were employed (Pearson: $r(55)=.236, p=.077$; Spearman: $r(55)=.189, p=.159)$. As in the total sample, associations between the BPQ and IATS were larger for individuals interpreting the BPQ as assessing attention than those interpreting it as assessing accuracy (Pearson: $Z=3.11, p<.001$; Spearman: $Z=2.46, p=.007$ ) and associations between the BPQ and IAS were larger for individuals interpreting the BPQ as assessing accuracy than those interpreting it as assessing attention (Pearson: $Z=-2.51$, $p=.006 ;$ Spearman: $Z=-2.46, p=.007)$.

In the total sample of individuals who interpreted the BPQ as assessing how often the signal objectively occurs within their body, a significant association was observed between the IATS and BPQ (Pearson: $r(115)=.374, p<.001$; Spearman: $r(55)=.353, p<.001)$. No association was observed between the IAS and BPQ (all $p>.250$ ) or the IAS and IATS (all $p>.250$ ). This pattern of significance was the same after the exclusion of individuals reporting mental health conditions (and those who declined to specify) or English as an additional language; an association was observed between the IATS and BPQ (all $p<.001$ ) and no association was observed between the IAS and BPQ (all $p>.10$ ) or the IATS and IAS (all 
$p>.250)$. Given that few individuals interpreted the BPQ as assessing 'other' $(\mathrm{N}=12)$

relationships between measures were not examined in this sample.

\subsubsection{Unplanned exploratory analyses}

For consistency with Study 1, we also examined associations excluding individuals with physical conditions (and those who declined to specify) in addition to those reporting mental health conditions (and those who declined to specify) and English as an additional language $(\mathrm{N}=213)$. Total sample correlations were similar to those reported above (see supplementary information [S11]). Whilst power was substantially reduced, a similar pattern also emerged within the separate sub-samples. In individuals reporting the BPQ as assessing attention, significant positive correlations were observed between the IATS and BPQ (Pearson: $r(54)=.560, p<.001$; Spearman: $r(54)=.493, p<.001)$. Correlations between the IAS and BPQ, were negative, although only significant using non-parametric analysis (Pearson: $r(54)=-.250$, $\mathrm{p}=.063$; Spearman: $r(54)=-.330, p=.013)$. No association was observed between the IAS and IATS (all $p>$.13). For those interpreting the BPQ as assessing accuracy, no correlation was observed between the IATS and BPQ (Pearson: $r(36)=.113, p>.250$; Spearman: $r(36)=.230$, $p=.164$ ), but a significant correlation was observed between the BPQ and IAS (Pearson: $r(36)=.338, p=.038$; Spearman: $r(36)=.353, p=.030)$. No association was observed between the IAS and IATS (all $p>.20$ ). Again, comparisons of the size of these correlations suggested that the size of the correlation between the IATS and BPQ was significantly larger in those who interpreted the measure as assessing attention, compared to accuracy, when parametric correlations were compared (Pearson: $Z=2.38, p<.01$; Spearman: $Z=1.40, p=.08$ ). The size of the correlation between the IAS and BPQ was also significantly larger in those who 
interpreted the BPQ as assessing accuracy, compared to those who interpreted the BPQ as assessing attention (Pearson: $Z=-2.79, p=.003$; Spearman: $Z=-3.27, p<.001$ ).

In Study 2, participants were also invited to provide feedback on the three questionnaires employed. Content analysis of these responses suggested that for the IATS, some participants felt the phrase 'most of the time' lacked clarity and that it was difficult to distinguish between the extent to which the signals objectively occurred and how much attention was focused on these signals. For the IAS, less consistent feedback was observed though participants generally commented on difficulty assessing their accuracy for some items. For the BPQ, participants commented on lack of clarity regarding the term 'awareness' as well as some instructional terms (e.g., focus, most situations) and response options (e.g., the similarity of occasionally and sometimes). For full details of feedback provided please see supplementary information [S12].

As in Study 1, we also examined possible non-linear associations between the measures of interoceptive accuracy and attention. Whilst evidence of non-linear associations was observed, these associations were not all consistent with Study 1; in the total sample, evidence of both quadratic and cubic trends were observed in the IAS and BPQ model. A quadratic (as in Study 1), but not cubic, trend was observed between the IAS and IATS. Where sub-samples are concerned, whilst a quadratic trend was observed between the IAS and IATS across sub-samples (though this was a non-significant trend in the group who interpreted the BPQ as assessing accuracy), for the BPQ an inconsistent pattern was observed; no non-linear trends were observed when individuals interpreted the BPQ as assessing attention, though significant quadratic trends were observed when individuals interpreted the BPQ as assessing accuracy or how often the signal objectively occurs (for details see supplementary information [S13]). 


\subsection{Discussion}

The results of Study 2 indicate that there is wide variation in participants' interpretation of the BPQ measure of self-reported interoception. Importantly, the interpretation of the BPQ altered associations between the BPQ and both the IATS and IAS; where individuals interpreted the BPQ as assessing accuracy, a relationship was generally found between the BPQ and IAS (a measure of accuracy) but not between the BPQ and the IATS (a measure of attention). In contrast, in those who believed the BPQ to be a measure of attention, relationships were found between the BPQ and IATS (a measure of attention) but not the BPQ and IAS (a measure of accuracy). Indeed, where relationships between the BPQ and IAS were observed these were negative, suggesting that greater self-reported attention (as assessed by the BPQ) related to lower self-reported accuracy. Overall, whilst more participants interpreted the BPQ as assessing attention, these data caution against the use of the BPQ as a pure measure of self-reported interoceptive attention. Notably, the majority of other self-reported interoception measures are limited by the fact that they include both items that assess interoceptive accuracy and those that assess interoceptive attention, which causes difficulties interpreting overall scores. The BPQ awareness scale, on the other hand, uses consistent wording across items, but interpretation still seems to be hampered by participants' own interpretation of the questionnaire. We therefore propose that using the IAS and IATS in combination will allow researchers to be confident that they are measuring self-reported interoceptive accuracy and attention separately.

\section{Study 3 (test-retest)}

Given that the results of Study 2 raise concerns regarding the utility of the BPQ as a pure measure of interoceptive attention, it is essential to establish the suitability of the IATS as a 
measure of interoceptive attention for future research. As such, Study 3 sought to examine the test-retest reliability of the IATS.

\subsection{Methods}

The test-retest reliability of the IATS was examined over a 28-day period in a third sample. As pre-registered (https://osf.io/rscgd), at Time 2, participants had an additional 7-days to complete the questionnaire giving a maximum time delay of 35 days between Time 1 and Time 2. This period of 28-35 days was set to be comparable to previous test-retest research for the BPQ and IAS (Murphy, Brewer, et al., 2020). As in Study 2, at the end of the questionnaire participants were asked how they interpreted the questionnaire and a free text box was given for further comments (see Study 2 for details). The questionnaire was completed online via Qualtrics (Provo, UT) and was automatically redistributed to participants 28 days after completion at Time 1. Participants were recruited via social media and pre-existing databases of individuals who had expressed an interest in taking part in psychological research. A prize draw was offered as an incentive. Data collection was set to continue until at least 100 participants had completed the questionnaire at both Time 1 and Time 2 (providing $>80 \%$ power to detect an effect size of $r=.25$ ). Having removed duplicate completions, incomplete responses and responses where participants completed Time 2 more than 7 days after the email request to complete Time 2 (>35 days after Time 1$)$, the final sample consisted of 146 participants $\left(M_{\mathrm{age}}=38.59\right.$ years, $S D_{\mathrm{age}}=15.88$ years, age range $18-77$ years, 114 female, 31 male, 1 other). Of these individuals, $27.4 \%$ reported pre-existing psychiatric conditions ( $0.7 \%$ declined to answer), $24.7 \%$ reported pre-existing physical health conditions ( $0.7 \%$ declined to answer) and $8.9 \%$ reported English as a second language. 


\subsection{Results}

\subsubsection{Planned analyses}

Prior to assessing the relationship between measures, assumptions of normality were checked using histograms and Kolmogorov-Smirnov tests and the data were checked for outliers. No outliers ( $>3 \mathrm{x}$ interquartile range) were present. Whilst no deviation from normality was observed at Time 1 ( $p>.05)$, slight deviation from normality was observed at Time 2 $(p=.038)$. As such, both parametric and non-parametric analyses are reported below.

On average participants completed the IATS at Time 229 days after Time $1(\mathrm{M}=28.77, \mathrm{SD}=$ 1.41, Range 28-35). Good test-retest reliability was found for the IATS (Pearson: $r(144)=.709, p<.001$; Spearman: $\left.r_{s}(144)=.765, p<.001\right)$ across this period. R-to-Z-tests were used (Cohen, \& Cohen, 1983; implemented via the quantpsy web implementation; Preacher, 2002) to compare the IATS test-retest reliability to the test-retest reliability of other selfreport measures of interoception reported in Murphy, Brewer, et al., (2020). Pearson correlations were used for these comparisons for consistency with previous reports, and all comparisons are two-tailed given that no directional predictions were made. No significant difference in test-retest reliability was found between the IATS and the IAS $(Z=-0.772$, $p>.250)$, or the IATS and the BPQ $(Z=0.387, p>.250)$. However, the ICQ showed greater test-retest reliability than the IATS $(Z=2.02, p=.04)$.

\subsubsection{Planned exploratory analyses}

The test-retest reliability of the IATS was also examined after excluding participants who did not interpret the IATS as assessing attention at both Time 1 and Time 2. Consistent with Study 2, at Time $176 \%$ of the sample interpreted the IATS as assessing attention, $7.5 \%$ interpreted the IATS as assessing accuracy, $12.3 \%$ interpreted the IATS as assessing how often the signal occurred and $4.1 \%$ selected 'other'. At Time $2,74.7 \%$ interpreted the IATS 
as assessing attention, $11 \%$ as accuracy, $11 \%$ as how often the signal occurred and $3.4 \%$ selected 'other'. $28.1 \%$ of the sample selected different options for their interpretation at Time 1 and Time 2. After these exclusions the final sample consisted of 95 participants $\left(M_{\text {age }}=37.01\right.$ years, $S D_{\text {age }}=15.30$ years, Age Range 18-77, 83 female, 11 male, 1 other). Removal of such participants did not change the overall pattern of results; good test-retest reliability was found for the IATS (Pearson: $r(93)=.693, p<.001$; Spearman: $r_{s}(93)=.735$, $p<.001)$. The test-retest reliability of the IATS and other self-report measures of interoception (reported in Murphy, Brewer et al., 2020) were again compared using R-to-Z tests. No significant difference in reliability was found between the IATS and the IAS $(Z=-0.917$, $p>.250)$, or the IATS and the BPQ $(Z=0.122, p>.250)$. However, the ICQ showed greater test-retest reliability than the IATS $(Z=2.034, p=.042)$.

Analyses were also re-run excluding individuals who reported mental health conditions (and those who declined to specify) or English as an additional language. In the total sample (not excluding those who misinterpreted the IATS), test-retest for the IATS was similar to that reported above (Pearson: $r(91)=.673, p<.001$; Spearman: $r_{s}(91)=.738, p<.001$ ). Again, the test-retest reliability for the IATS was similar to that for the IAS and BPQ (both $p>.20$ ) but was lower than the ICQ ( $p=.022)$. This pattern of results held when inclusion was restricted to those who interpreted the IATS as assessing attention at both Time 1 and Time 2.

\subsubsection{Unplanned exploratory analyses}

For consistency with Study 1 and Study 2, we also examined associations excluding individuals with physical conditions (and those who declined to specify) in addition to those reporting mental health conditions (and those who declined to specify) and English as an additional language. Results were consistent with the above samples. Results were also 
consistent when inclusion was restricted to those who interpreted the IATS as assessing attention at both Time 1 and Time 2 in this subsample.

\subsection{Discussion}

The IATS was found to have good test-retest reliability, comparable to that of the IAS and BPQ (although lower than that of the ICQ). These results were replicated having excluded participants who did not correctly interpret the IATS as assessing attention, those reporting mental or physical health conditions, and those with English as an additional language.

\section{General discussion}

The aim of this series of studies was to test the proposed distinction between self-reported accuracy and self-reported attention (Murphy, Brewer, et al., 2020; Murphy et al., 2019). Overall, results are consistent with separation of self-reported accuracy and attention; across both Study One and Study Two, relationships were observed between self-report measures of interoceptive attention and between self-report measures of accuracy. Little evidence was observed for relationships between measures assessing different facets of interoception (accuracy or attention), and where these relationships were observed, they were substantially smaller in size than those between measures of the same facet of interoception. Where relationships across facets were observed, these often suggested a small inverse relationship between self-reported accuracy and attention, whereby greater self-reported attention was related to poorer self-reported accuracy. In Study Two, we also demonstrated the presence of individual differences in the interpretation of questionnaire measures of interoception, particularly the BPQ (Porges, 1993). Importantly, the interpretation of this measure altered associations between the BPQ and other questionnaire measures of interoception.

The results of Study One and Study Two are consistent with the proposal that self-reported interoceptive accuracy and self-reported interoceptive attention are different constructs 
(Murphy, Brewer, et al., 2020; Murphy et al., 2019). Indeed, as the interoceptive signals included in the IATS were specifically designed to match those in the IAS, the lack of a linear association between the IAS (a measure of accuracy) and IATS (a measure of attention) suggests that previous reports of a lack of an association between the IAS and BPQ are not due to differences in the sensations rated across these questionnaires (Murphy, Brewer, et al., 2020). Given previous evidence that measures of self-reported interoceptive attention and self-reported interoceptive accuracy may be differentially associated with psychopathology (Murphy, Brewer, et al., 2020) these results underscore the importance of considering which specific facet of interoception is quantified by measures of interoception, at least for self-report measures. Future research examining whether this dissociation extends to objectively measured facets of interoception, or indeed other aspects of interoception (e.g., propensity to utilise signals, magnitude of signals; Khalsa et al., 2018; Murphy, Catmur, \& Bird, 2018), is warranted. This is likely to be important for research in clinical groups, where different facets of interoception may relate differentially to mental health, developmental research, as different facets of interoception may follow different developmental trajectories, and work on typical cognition, as different interoceptive facets are likely to relate differently to abilities such as social and emotional abilities and decision-making.

Results from Study Two raise concerns regarding the interpretation of measures of selfreported interoception. For the BPQ in particular, no consensus in interpretation of the term 'awareness' was observed. While the most common interpretation ( $\sim 36 \%$ of participants) was that the BPQ was assessing interoceptive attention, a high number of participants believed that it was assessing interoceptive accuracy, the objective frequency of interoceptive signals, or otherwise. Importantly, results revealed that the interpretation of the BPQ altered associations between the BPQ and other questionnaire measures of interoception. In individuals who interpreted the BPQ as assessing attention, a relationship between the BPQ 
and IATS (a measure of attention) was observed, but no relationship was observed between the BPQ and IAS (a measure of accuracy). In individuals who interpreted the BPQ as assessing accuracy, the opposite pattern of results was generally found, though it should be noted that this relationship was less reliable than the relationship between the IATS and BPQ within individuals who interpreted the BPQ as assessing attention. Whilst it is unclear why this may be, in light of evidence that objective interoceptive accuracy may dissociate across domains (Ferentzi, Bogdány, et al., 2018; Garfinkel, Manassei, et al., 2016; Steptoe \& Vögele, 1992; but see Herbert et al., 2012; Whitehead \& Drescher, 1980), and the fact that the BPQ examines different interoceptive signals than the IAS and IATS, it is possible to speculate that self-reported interoceptive accuracy may differ depending on the interoceptive signals assessed within questionnaires, whereas self-reported interoceptive attention may generalise across interoceptive domains. Regardless of this, given frequent use of the BPQ in the literature as a measure of self-reported interoception and for the calculation of other facets of interoception (e.g., Garfinkel, Tiley, et al., 2016; Palser et al., 2018), these data hold important implications suggesting that differential results may be observed across studies due to differences in the interpretation of the measure across samples. It is of note that the BPQ is often used as a general measure of interoceptive sensibility (self-reported interoception, not specifying accuracy or attention), but the current findings and previous work (Murphy, Brewer, et al., 2020) suggest that this is an important distinction to make. Utilising tools that are less ambiguous in their interpretation, and can be seen as pure measures of interoceptive accuracy or interoceptive attention, is therefore likely to make interpretation of findings easier. However, it is important to acknowledge that not all individuals interpreted the IAS and IATS as measures of accuracy and attention, respectively, and in Study Three some evidence of differences within individuals was observed. As such, future research examining 
self-reported interoception should include measures that assess individuals' interpretations of these questionnaires, and efforts should be made to improve clarity of instructions.

Given concerns over the BPQ as a measure of self-reported interoceptive attention, the IATS may be a useful tool for examining self-reported interoceptive attention. Initial evidence suggests that this measure is more likely to be interpreted as assessing attention than the $\mathrm{BPQ}$, with an additional advantage being that the interoceptive signals included are directly matched to those assessed by the IAS. The use of these two questionnaires in combination, therefore, allows for specific investigation of self-reported abilities across the two facets of interoceptive accuracy and interoceptive attention, without discrepancies between the measures being potentially attributed to different interoceptive signals. The IATS appears to have good internal consistency and its test-retest reliability is comparable to existing measures of self-reported interoception. Whilst more research is required to further examine the factor structure of the measure, for investigations requiring separate assessment of interoceptive accuracy and attention, total scores from the IAS and IATS would allow comparisons using measures that assess the same interoceptive signals.

Whilst the above results were in line with our pre-registered predictions, it should be noted that some evidence of an inverse relationship between self-reported interoceptive attention and accuracy was observed, whereby greater attention was associated with lower accuracy. Such an association is perhaps unsurprising, as individuals who believe their interoceptive accuracy to be low may pay more attention to internal signals in an attempt to compensate for poor accuracy and recognise the sensation. Although this inverse relationship supports dissociation of these constructs (i.e., that they are not interchangeable), further examination of the relationship between self-reported interoceptive accuracy and self-reported interoceptive attention is required to understand whether such a pattern is clinically meaningful. Likewise, future research should examine the possibility of non-linear 
associations between these constructs. Although some evidence of a small quadratic association between self-reported accuracy and attention was observed, the observation of no quadratic association in individuals who interpreted the BPQ as assessing attention requires further scrutiny. Whilst relationships between the IATS and IAS are consistent with the possibility of a non-linear relationship between accuracy and attention, further research is required to establish the reliability of these effects and to understand what is driving this association. In order to accurately investigate these relationships further, it is essential that pure measures of interoceptive accuracy and attention are utilised.

Despite the utility of these data for our understanding of the relationship between measures of self-reported interoception, limitations must be acknowledged. First, although online testing is increasingly common in psychological research, there are inherent limitations to this approach (e.g., generalisability, control of environment). Second, as these data were collected during the COVID-19 pandemic, it is not possible to rule out a contribution of the present climate to these results. Third, whilst efforts were made to construct the IATS instructions to ensure separation of interoceptive attention and how often the signal objectively occurs, it should be acknowledged that such a separation may not always be applicable; for example, whilst an individual may often pay attention to whether they need to urinate (regardless of whether the sensation objectively occurs), for some individuals attention may be strictly bound to the presence of these signals. The role of the presence of the signal on attention may also depend on the signal, for example attention to pain being more closely associated with the presence of a painful stimulus, but the attention to heart rate being more consistent across contexts, and again these attentional profiles may differ across individuals. Further research is therefore required to understand the extent to which these aspects of interoception are linked or dissociate across individuals, and dissociate across interoceptive signals. 
In conclusion, results from this series of studies suggest dissociations between self-reported interoceptive accuracy and self-reported interoceptive attention, which remain even when the to-be-rated interoceptive signals are matched across questionnaires. Variation in participants' interpretation of the BPQ caution against reliance on the BPQ as a measure of self-reported interoceptive attention, and suggest a need for greater consideration of the interpretation of questionnaire measures of interoception. The current study also provides initial support for the use of a novel measure of self-reported interoceptive attention, the IATS, which appears to be a valid measure of self-reported interoceptive attention, and is matched in interoceptive signals to the existing IAS measure of self-reported interoceptive accuracy, allowing for comparison of these two distinct facets of interoception. 


\section{Acknowledgements}

RB is supported by a New Investigator Grant from the Medical Research Council. 


\section{References}

Ardizzi, M., Ambrosecchia, M., Buratta, L., Ferri, F., Peciccia, M., Donnari, S., ... Gallese, V. (2016). Interoception and positive symptoms in schizophrenia. Frontiers in Human Neuroscience, 10, 379. doi:10.3389/fnhum.2016.00379

Bechara, A., \& Damasio, A. R. (2005). The somatic marker hypothesis: A neural theory of economic decision. Games and economic behavior, 52(2), 336-372. doi:10.1016/j.geb.2004.06.010

Brener, J., \& Ring, C. (2016). Towards a psychophysics of interoceptive processes: the measurement of heartbeat detection. Philosophical Transactions of the Royal Society B: Biological Sciences, 371(1708), 20160015.

Brewer, R., Cook, R., \& Bird, G. (2016). Alexithymia: a general deficit of interoception. Royal Society Open Science, 3(10), 150664. doi:10.1098/rsos.150664

Campos, C., Murphy, J., Brewer, R., Gabriele, E., Rocha, N., \& Barbosa, F. (2020, July 3). Quadratic Association Between Self-Reported Interoceptive Accuracy and Attention. Retrieved from osf.io/v632y

Cohen, J., \& Cohen, P. (1983). Applied multiple regression/correlation analysis for the behavioral sciences. Hillsdale, NJ: Erlbaum.

Critchley, H. D., \& Harrison, N. A. (2013). Visceral influences on brain and behavior. Neuron, 77(4), 624-638. doi:10.1016/j.neuron.2013.02.008

Damasio, A. R. (1994). Descartes' error: Emotion, rationality and the human brain.

Dunn, B. D., Galton, H. C., Morgan, R., Evans, D., Oliver, C., Meyer, M., ... Dalgleish, T. (2010). Listening to your heart. How interoception shapes emotion experience and intuitive decision making. Psychological Science, 21(12), 1835-1844. doi:10.1177/0956797610389191

Eggart, M., Lange, A., Binser, M. J., Queri, S., \& Müller-Oerlinghausen, B. (2019). Major depressive disorder is associated with impaired interoceptive accuracy: A systematic review. Brain sciences, 9(6), 131.

Ferentzi, E., Bogdány, T., Szabolcs, Z., Csala, B., Horváth, Á., \& Köteles, F. (2018). Multichannel investigation of interoception: sensitivity is not a generalizable feature. Frontiers in Human Neuroscience, 12, 223. doi:10.3389/fnhum.2018.00223

Ferentzi, E., Drew, R., Tihanyi, B. T., \& Köteles, F. (2018). Interoceptive accuracy and body awareness - Temporal and longitudinal associations in a non-clinical sample. Physiology \& Behavior, 184, 100-107. doi:10.1016/j.physbeh.2017.11.015

Field, A. (2005). Discovering statistics with SPSS.

Forkmann, T., Scherer, A., Meessen, J., Michal, M., Schächinger, H., Vögele, C., \& Schulz, A. (2016). Making sense of what you sense: Disentangling interoceptive awareness, sensibility and accuracy. International Journal of Psychophysiology, 109, 71-80. doi:10.1016/j.ijpsycho.2016.09.019 
Garfinkel, S. N., Barrett, A. B., Minati, L., Dolan, R. J., Seth, A. K., \& Critchley, H. D. (2013). What the heart forgets: Cardiac timing influences memory for words and is modulated by metacognition and interoceptive sensitivity. Psychophysiology, 50(6), 505-512. doi:10.1111/psyp.12039

Garfinkel, S. N., Manassei, M. F., Hamilton-Fletcher, G., In den Bosch, Y., Critchley, H. D., \& Engels, M. (2016). Interoceptive dimensions across cardiac and respiratory axes. Philosophical Transactions of the Royal Society of London. Series B, Biological Sciences, 371(1708). doi:10.1098/rstb.2016.0014

Garfinkel, S. N., Seth, A. K., Barrett, A. B., Suzuki, K., \& Critchley, H. D. (2015). Knowing your own heart: distinguishing interoceptive accuracy from interoceptive awareness. Biological Psychology, 104, 65-74. doi:10.1016/j.biopsycho.2014.11.004

Garfinkel, S. N., Tiley, C., O’Keeffe, S., Harrison, N. A., Seth, A. K., \& Critchley, H. D. (2016). Discrepancies between dimensions of interoception in autism: Implications for emotion and anxiety. Biological Psychology, 114, 117-126. doi:10.1016/j.biopsycho.2015.12.003

Herbert, B. M., Muth, E. R., Pollatos, O., \& Herbert, C. (2012). Interoception across modalities: on the relationship between cardiac awareness and the sensitivity for gastric functions. Plos One, 7(5), e36646. doi:10.1371/journal.pone.0036646

Khalsa, S. S., Adolphs, R., Cameron, O. G., Critchley, H. D., Davenport, P. W., Feinstein, J. S., ... Interoception Summit 2016 participants. (2018). Interoception and mental health: A roadmap. Biological Psychiatry: Cognitive Neuroscience and Neuroimaging, 3(6), 501-513. doi:10.1016/j.bpsc.2017.12.004

Lee, I. A., \& Preacher, K. J. (2013). Calculation for the test of the difference between two dependent correlations with no variable in common [Computer software]. Available from http://quantpsy.org.

Lee, I. A., \& Preacher, K. J. (2013). Calculation for the test of the difference between two dependent correlations with one variable in common [Computer software]. Available from http://quantpsy.org.

Löffler, A., Foell, J., \& Bekrater-Bodmann, R. (2018). Interoception and Its Interaction with Self, Other, and Emotion Processing: Implications for the Understanding of Psychosocial Deficits in Borderline Personality Disorder. Current Psychiatry Reports, 20(4), 28. doi:10.1007/s11920-018-0890-2

Murphy, J., Brewer, R., Catmur, C., \& Bird, G. (2017). Interoception and psychopathology: A developmental neuroscience perspective. Developmental cognitive neuroscience, 23, 45-56. doi:10.1016/j.den.2016.12.006

Murphy, J., Brewer, R., Plans, D., Khalsa, S. S., Catmur, C., \& Bird, G. (2020). Testing the independence of self-reported interoceptive accuracy and attention. Quarterly Journal of Experimental Psychology, 73(1), 115-133.

Murphy, J., Catmur, C., \& Bird, G. (2018). Alexithymia is associated with a multidomain, multidimensional failure of interoception: Evidence from novel tests. Journal of Experimental Psychology: General, 147(3), 398-408. doi:10.1037/xge0000366 
Murphy, J., Catmur, C., \& Bird, G. (2019). Classifying individual differences in interoception: Implications for the measurement of interoceptive awareness. Psychonomic Bulletin \& Review, 26(5), 1467-1471. doi:10.3758/s13423-019-01632-7

O'Connor, B. P. (2000). SPSS and SAS programs for determining the number of components using parallel analysis and velicer's MAP test. Behavior research methods, instruments, \& computers : a journal of the Psychonomic Society, Inc, 32(3), 396402.

Palser, E. R., Fotopoulou, A., Pellicano, E., \& Kilner, J. M. (2018). The link between interoceptive processing and anxiety in children diagnosed with autism spectrum disorder: Extending adult findings into a developmental sample. Biological Psychology, 136, 13-21. doi:10.1016/j.biopsycho.2018.05.003

Paulus, M. P., \& Stein, M. B. (2010). Interoception in anxiety and depression. Brain Structure \& Function, 214(5-6), 451-463. doi:10.1007/s00429-010-0258-9

Pituch, K. A., \& Stevens, J. P. (2015). Applied multivariate statistics for the social sciences: Analyses with SAS and IBM's SPSS. taylorfrancis.com.

Pollatos, O., \& Schandry, R. (2008). Emotional processing and emotional memory are modulated by interoceptive awareness. Cognition \& emotion, 22(2), 272-287. doi:10.1080/02699930701357535

Pollatos, O., Kurz, A.-L., Albrecht, J., Schreder, T., Kleemann, A. M., Schöpf, V., ... Schandry, R. (2008). Reduced perception of bodily signals in anorexia nervosa. Eating Behaviors, 9(4), 381-388. doi:10.1016/j.eatbeh.2008.02.001

Porges, S. (1993). Body perception questionnaire. Laboratory of Developmental Assessment, University of Maryland.

Preacher, K. J. (2002). Calculation for the test of the difference between two independent correlation coefficients [Computer software]. Available from http://quantpsy.org.

Schultchen, D., Zaudig, M., Krauseneck, T., Berberich, G., \& Pollatos, O. (2019). Interoceptive deficits in patients with obsessive-compulsive disorder in the time course of cognitive-behavioral therapy. Plos One, 14(5), e0217237. doi:10.1371/journal.pone.0217237

Schulz, A., Lass-Hennemann, J., Sütterlin, S., Schächinger, H., \& Vögele, C. (2013). Cold pressor stress induces opposite effects on cardioceptive accuracy dependent on assessment paradigm. Biological Psychology, 93(1), 167-174. doi:10.1016/j.biopsycho.2013.01.007

Sönmez, M. B., Kahyacı Kılıç, E., Ateş Çöl, I., Görgülü, Y., \& Köse Çınar, R. (2017). Decreased interoceptive awareness in patients with substance use disorders. Journal of substance use, 22(1), 60-65. doi:10.3109/14659891.2016.1143048

Steiger, J. H. (1980). Tests for comparing elements of a correlation matrix. Psychological Bulletin, 87(2), 245-251. doi:10.1037/0033-2909.87.2.245 
Steptoe, A., \& Vögele, C. (1992). Individual differences in the perception of bodily sensations: the role of trait anxiety and coping style. Behaviour Research and Therapy, 30(6), 597-607. doi:10.1016/0005-7967(92)90005-2

Sugawara, A., Terasawa, Y., Katsunuma, R., \& Sekiguchi, A. (2020). Effects of interoceptive training on decision making, anxiety, and somatic symptoms. BioPsychoSocial medicine, 14, 7. doi:10.1186/s13030-020-00179-7

Terasawa, Y., Fukushima, H., \& Umeda, S. (2013). How does interoceptive awareness interact with the subjective experience of emotion? An fMRI study. Human Brain Mapping, 34(3), 598-612. doi:10.1002/hbm.21458

Werner, N. S., Peres, I., Duschek, S., \& Schandry, R. (2010). Implicit memory for emotional words is modulated by cardiac perception. Biological Psychology, 85(3), 370-376. doi:10.1016/j.biopsycho.2010.08.008

Whitehead, W. E., \& Drescher, V. M. (1980). Perception of Gastric Contractions and SelfControl of Gastric Motility. Psychophysiology, 17(6), 552-558. doi:10.1111/j.14698986.1980.tb02296.x

Zamariola, G., Frost, N., Van Oost, A., Corneille, O., \& Luminet, O. (2019). Relationship between interoception and emotion regulation: New evidence from mixed methods. Journal of Affective Disorders, 246, 480-485. doi:10.1016/j.jad.2018.12.101 


\section{Supplementary information}

\section{[S1] Psychometric properties of the IATS}

Cronbach's alpha indicated good internal consistency for the IATS $(\alpha=.905)$ that was slightly lower than the other measure of interoceptive attention (the BPQ: $\alpha=.971$ ) but higher than the measures of interoceptive accuracy (IAS: $\alpha=.880$; ICQ: $\alpha=.510$ ). Prior to examining the factor structure of the IATS, the suitability of the IATS for analysis was examined. First, correlational analyses revealed that all 21 IATS items correlated with at least one other item. Second, Kaiser-Meyer-Olkin measure of sampling adequacy was .917, above the commonly recommended value of $~ .5$, and Bartlett's test of sphericity was significant, $\chi 2(210)=4978.714, p<.001$. The diagonals of the anti-image correlation matrix were all $\geq$ .825 . Finally, the communalities were all $\geq .292$. Thurs, the data was deemed to be suitable for factor analysis.

PCA with Direct Oblimin rotation (Field, 2005) was used to examine the factor structure with coefficients $<.40$ suppressed (Pituch \& Stevens, 2015). Examination of the scree plot and parallel analysis (implemented using an existing SPSS routine from O’Connor, 2000) was used to determine the number of factors to extract. This indicated a 3 -factor solution for the data (Figure [S2]) explaining 36.24\%, 7.12\% and 6.39\% of the variance, respectively. Three items, taste, sexual arousal, and muscular fatigue did not load onto any of these factors at the threshold of $>.40$ but loaded $>.39$. Whilst there does not appear to be a clear explanation for these groupings, Factor 1 seems to reflect attention to various physiological sensations, Factor 2 appears to comprise items that relate to alimentary signals (Thirst, Hunger, Urination, Defecation, Wind) and Factor 3 appears to comprise mostly sensations that may be perceived via the skin (Affective Touch, Tickle, Pain from injury, Temperature, Taste). An exploratory factor analysis (EFA) using Direct Oblimin rotation was also conducted. Given 
that parallel analysis may over extract factors for EFA, extraction was set to 3 factors as in the PCA. This three-factor solution explained $33.72 \%, 4.50 \%$ and $3.67 \%$ of the variance, respectively. The EFA produced a broadly similar pattern of factor loadings for most factors, though more items failed to reach the loading threshold (Figure [S3]).

[S2] Principal components analysis pattern matrix

\begin{tabular}{|c|c|c|c|}
\hline Vomit & $\begin{array}{c}\text { Factor } 1 \\
\mathbf{0 . 7 6 8}\end{array}$ & Factor 2 & Factor 3 \\
\hline Sneeze & 0.760 & & \\
\hline Cough & 0.753 & & \\
\hline Breathe & 0.685 & & \\
\hline Burp & 0.662 & & \\
\hline Heart & 0.628 & & \\
\hline Pain (no injury) & 0.540 & & \\
\hline Itch & 0.532 & & \\
\hline Wind & 0.516 & -0.392 & \\
\hline Blood sugar & 0.407 & & \\
\hline Sexual arousal & 0.392 & & \\
\hline Thirst & & -0.765 & \\
\hline Hunger & & -0.725 & \\
\hline Urinate & & -0.670 & \\
\hline Defecate & & -0.592 & \\
\hline Affective touch & & & 0.683 \\
\hline Tickle & 0.493 & & 0.506 \\
\hline Pain (with injury) & & & 0.489 \\
\hline Temperature & & & 0.412 \\
\hline Tired muscles & & & 0.394 \\
\hline Taste & & & 0.391 \\
\hline
\end{tabular}

Figure [S2]. Pattern matrix for the PCA with Direct Oblimin Rotation. Items are arranged by their magnitude of factor loading. Bold items loaded above the pre-defined cut-off of .40, with items that loaded above .39 indicated by non-bolded text. 
[S3] Exploratory factor analysis pattern matrix

\begin{tabular}{|c|c|c|c|}
\hline & & Factor 2 & Factor 3 \\
\hline Sneeze & 0.808 & & \\
\hline Cough & 0.780 & & \\
\hline Burp & 0.690 & & \\
\hline Vomit & 0.687 & & \\
\hline Wind & 0.510 & & \\
\hline Breathe & 0.451 & & \\
\hline \multicolumn{4}{|l|}{ Heart } \\
\hline \multicolumn{4}{|l|}{ Sexual arousal } \\
\hline \multicolumn{4}{|l|}{ Blood sugar } \\
\hline Thirst & & -0.683 & \\
\hline Urinate & & -0.607 & \\
\hline Hunger & & -0.582 & \\
\hline Defecate & 0.419 & -0.506 & \\
\hline Tickle & & & 0.586 \\
\hline Pain (injury) & & & 0.504 \\
\hline Itch & & & 0.466 \\
\hline Pain (noinjury) & & & 0.446 \\
\hline Affective touch & & & 0.445 \\
\hline Tired muscles & & & 0.436 \\
\hline Temperature & & & 0.399 \\
\hline Taste & & & \\
\hline
\end{tabular}

Figure [S3]. Pattern matrix for the EFA with Direct Oblimin Rotation. Items are arranged by their magnitude of factor loading. Bold items loaded above the pre-defined cut-off of.40, with items that loaded above .39 indicated by non-bolded text. 
[S4] Table of R-to-Z tests for Study 1 , full sample

\begin{tabular}{|l|l|l|l|l|l|}
\hline & \multicolumn{3}{|l|}{ Pearson } & \multicolumn{2}{l|}{ Spearman } \\
\hline Correlation A & Correlation B & $\mathbf{Z}$ & $\mathbf{P}$ & $\mathbf{Z}$ & $\mathbf{P}$ \\
\hline IAS-ICQ & BPQ-IAS & 12.26 & $<.001$ & 11.89 & $<.001$ \\
\hline IAS-ICQ & BPQ-ICQ & 14.59 & $<.001$ & 14.58 & $<.001$ \\
\hline IAS-ICQ & IATS-IAS & 12.64 & $<.001$ & 12.40 & $<.001$ \\
\hline IAS-ICQ & IATS-ICQ & 15.77 & $<.001$ & 15.55 & $<.001$ \\
\hline BPQ-IATS & BPQ-IAS & 5.48 & $<.001$ & 6.55 & $<.001$ \\
\hline BPQ-IATS & BPQ-ICQ & 6.68 & $<.001$ & 7.96 & $<.001$ \\
\hline BPQ-IATS & IATS-IAS & 6.39 & $<.001$ & 7.57 & $<.001$ \\
\hline BPQ-IATS & IATS-ICQ & 8.23 & $<.001$ & 9.37 & $<.001$ \\
\hline
\end{tabular}

All $\mathrm{p}$ values are 1-tailed. 
[S5] Correlations and R-to-Z tests between measures in Study 1 excluding individuals with mental health conditions (and those who declined to answer this question) and English as an additional language

\begin{tabular}{llllllllll}
\hline $\begin{array}{l}\text { Pearson } \\
\text { Correlations }\end{array}$ & \multicolumn{7}{c}{$\begin{array}{l}\text { Spearman } \\
\text { Correlations }\end{array}$} \\
\hline \multicolumn{2}{c}{ IATS } & IAS & BPQ & ICQ & & IATS & IAS & BPQ & ICQ \\
IAS & $-.121 *$ & 1 & & & IAS & $-.180 * *$ & 1 & & \\
BPQ & $.330 * *$ & .047 & 1 & & BPQ & $.364 * *$ & -.009 & 1 & \\
ICQ & $.217 * *$ & $-.565^{* *}$ & .11 & 1 & ICQ & $.263 * *$ & $-.579 * *$ & $.156 * *$ & 1 \\
\hline
\end{tabular}

$* *$ denotes significant at $\mathrm{p}<.001 *$ denotes significant at $\mathrm{p}<.05$ (two-tailed)

\begin{tabular}{|l|l|l|l|l|l|}
\hline & & \multicolumn{2}{l|}{ Pearson } & \multicolumn{2}{l|}{ Spearman } \\
\hline Correlation A & Correlation B & $\mathbf{Z}$ & $\mathbf{P}$ & $\mathbf{Z}$ & $\mathbf{P}$ \\
\hline IAS-ICQ & BPQ-IAS & 6.88 & $<.001$ & 7.67 & $<.001$ \\
\hline IAS-ICQ & BPQ-ICQ & 9.60 & $<.001$ & 10.24 & $<.001$ \\
\hline IAS-ICQ & IATS-IAS & 8.60 & $<.001$ & 9.41 & $<.001$ \\
\hline IAS-ICQ & IATS-ICQ & 10.27 & $<.001$ & 10.88 & $<.001$ \\
\hline BPQ-IATS & BPQ-IAS & 3.42 & $<.001$ & 4.42 & $<.001$ \\
\hline BPQ-IATS & BPQ-ICQ & 5.09 & $<.001$ & 5.96 & $<.001$ \\
\hline BPQ-IATS & IATS-IAS & 5.91 & $<.001$ & 7.01 & $<.001$ \\
\hline BPQ-IATS & IATS-ICQ & 6.68 & $<.001$ & 7.60 & $<.001$ \\
\hline
\end{tabular}

All $\mathrm{p}$ values are 1-tailed. 
[S6] Correlations and R-to-Z tests comparing relationships between measures in Study 1 excluding individuals with mental health conditions (and those who declined to answer this question), physical health conditions (and those who declined to answer this question) and English as an additional language

\begin{tabular}{|c|c|c|c|c|c|c|c|c|c|}
\hline \multicolumn{5}{|c|}{$\begin{array}{l}\text { Pearson } \\
\text { Correlations }\end{array}$} & \multicolumn{5}{|c|}{$\begin{array}{l}\text { Spearman } \\
\text { Correlations }\end{array}$} \\
\hline & IATS & IAS & $\overline{B P Q}$ & ICQ & & IATS & IAS & BPQ & ICQ \\
\hline IAS & -.100 & 1 & & & IAS & $-.170 * *$ & 1 & & \\
\hline BPQ & $.341 * *$ & .108 & 1 & & BPQ & $.357 * *$ & .063 & 1 & \\
\hline ICQ & $.213 * *$ & $-.523^{* *}$ & .081 & 1 & ICQ & $.257 * *$ & $-.539 * *$ & .115 & 1 \\
\hline
\end{tabular}

**denotes significant at $\mathrm{p}<.001 *$ denotes significant at $\mathrm{p}<.05$ (two-tailed)

\begin{tabular}{|l|l|l|l|l|l|}
\hline & \multicolumn{3}{|l|}{ Pearson } & \multicolumn{2}{l|}{ Spearman } \\
\hline Correlation A & Correlation B & Z & P & Z & P \\
\hline IAS-ICQ & BPQ-IAS & 4.85 & $<.001$ & 5.49 & $<.001$ \\
\hline IAS-ICQ & BPQ-ICQ & 7.67 & $<.001$ & 8.16 & $<.001$ \\
\hline IAS-ICQ & IATS-IAS & 6.76 & $<.001$ & 7.61 & $<.001$ \\
\hline IAS-ICQ & IATS-ICQ & 8.43 & $<.001$ & 8.92 & $<.001$ \\
\hline BPQ-IATS & BPQ-IAS & 2.51 & $<.01$ & 3.09 & $<.01$ \\
\hline BPQ-IATS & BPQ-ICQ & 4.31 & $<.001$ & 4.75 & $<.001$ \\
\hline BPQ-IATS & IATS-IAS & 5.27 & $<.001$ & 6.19 & $<.001$ \\
\hline BPQ-IATS & IATS-ICQ & 6.05 & $<.001$ & 6.66 & $<.001$ \\
\hline
\end{tabular}

All $\mathrm{p}$ values are 1-tailed. 


\section{[S7] Study 1: Examination of non-linear associations}

When non-linear associations between measures were investigated in the total sample, with accuracy as the dependent variable and attention as the independent variable (Campos et al., in prep), non-linear trends were observed between the IAS and BPQ. In terms of $\mathrm{R}^{2}$ change, the inclusion of the quadratic term (in addition to the linear term) significantly improved the regression model $(p<.001)$ whilst the inclusion of a cubic term only marginally improved the model $(p=.072)$. For the ICQ and BPQ, the inclusion of both the quadratic $(p<.001)$ and cubic ( $p=.024)$ terms improved the model fit. For the IAS and IATS, the inclusion of the quadratic $(p<.001)$ but not cubic $(p>.20)$ term significantly improved the model fit. For the ICQ and IATS, the inclusion of the quadratic term $(p<.001)$ significantly improved the model fit, whilst only marginal improvement was observed for the cubic trend ( $p=.078)$. In all models, the quadratic term indicated that higher self-reported accuracy was associated with both higher and lower levels of self-reported interoceptive attention. Cubic trends were difficult to interpret.

[S8] Interpretation question and response options for the BPQ, IAS and IATS for Study

\section{2 (IATS question also used in Study 3)}

1. BPQ: In this questionnaire we asked you to tell us how aware you are of certain sensations during most situations. While you were completing the questionnaire, what did you think the term 'aware' meant in this context? Please select one option that best describes your interpretation of the questionnaire.

2. IATS: In this questionnaire we asked you to tell us how much attention you pay to specific bodily sensations. While you were completing the questionnaire, what did 
you think the term 'attention' meant in this context? Please select one option that best describes your interpretation of the questionnaire.

3. IAS: In this questionnaire we asked you to tell us how accurately you can perceive specific bodily sensations. While you were completing the questionnaire, what did you think the term 'accuracy' meant in this context? Please select one option that best describes your interpretation of the questionnaire.

a. How much attention you pay to these sensations (e.g., how much they occupy your thoughts/mind, how much you think about these sensations, how much you monitor whether these signals are occurring or not (regardless of how well you can perceive them or how often they occur)).

b. How accurate you are at perceiving these sensations (e.g., how good you are at feeling/detecting them when they occur, how well you can tell them apart from other sensations when they occur, how precise you are at sensing them (regardless of how much you monitor them or how often they occur).

c. How often (frequently) or intensely these sensations actually occur in your body (e.g. how often your body is objectively cold, how often your muscles are objectively tired (regardless of how much you monitor them or how good you are at perceiving them).

d. None of the above. Please tell us.... 
[S9] Verbatim 'other' responses for BPQ interpretations from Study 2

- Actually a mixture of all of the above.

- Both a and b (attention and accuracy).

- Do I notice myself doing the above reactions in everyday life

- How aware of these sensations I am

- How conscious you are of a sensation, which may or may not be the same as how often the sensation actually occurs in your body (e.g. my lip trembles 4 times in a minute but I am conscious of this action occurring only once).

- How often I am aware of these sensations, regardless of the physical state of my body

- How often I notice them (if/when they occur).

- How often I notice them when they happen

- How often I pay attention to them when they occur

- How often you perceive these signals in a normal situation with distractions

- I answered with two thoughts in mind. Do I even have that feeling very often? If not then how can I be in tune with it. The more I am confronted by a certain stimulus, the more I am in tune with it.

- No interpretation specified by one participant

[S10] Correlations between measures in Study 2 excluding individuals with mental health conditions and English as an additional language

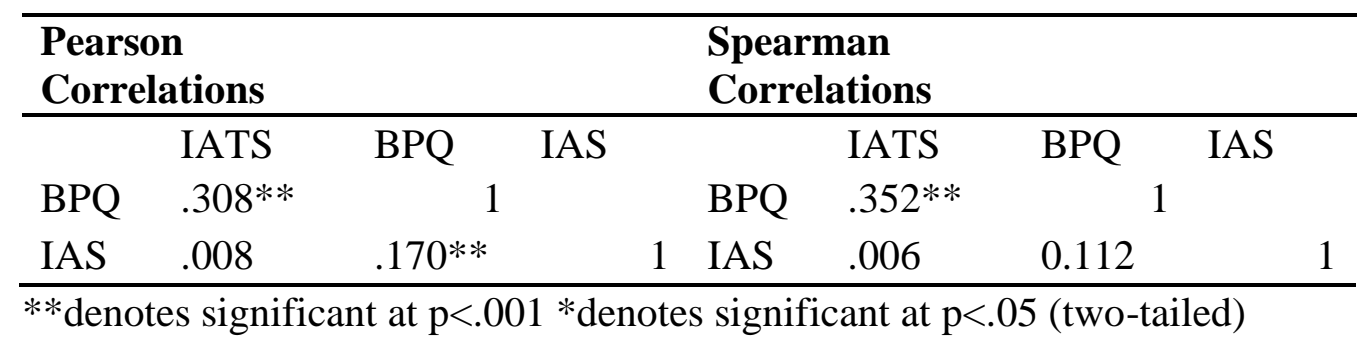

[S11] Correlations between measures in Study 2 excluding individuals with mental health conditions, physical health conditions and English as an additional language

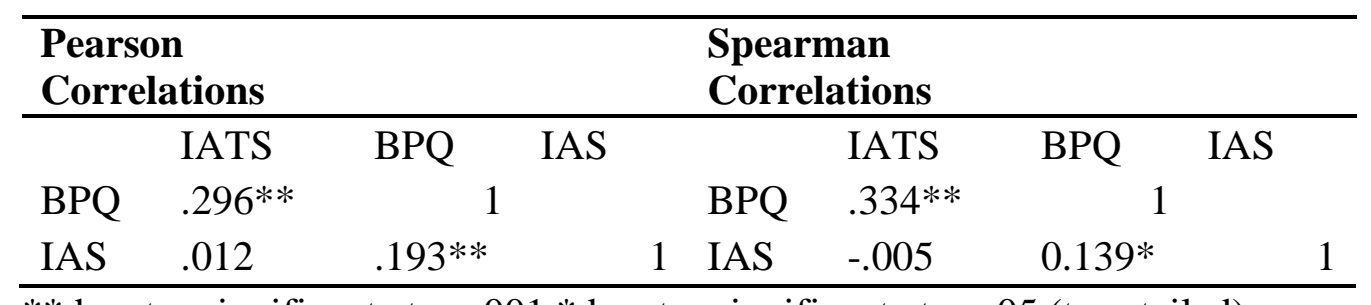

$* *$ denotes significant at $\mathrm{p}<.001 *$ denotes significant at $\mathrm{p}<.05$ (two-tailed) 


\section{[S12] Feedback about questionnaire measures from Study 2}

Answers of none, N/A and No have been removed. Typographical errors have been corrected and feedback unrelated to the questionnaire (e.g. providing additional demographic information, suggesting hypotheses/theories relating to the research, or thanking the authors for conducting the research) and/or uninterpretable responses have been removed.

\section{IATS}

- "most of the time" my attention is on a variety of things. I don't think about any one thing "most of the time"

- Confusing wording, had to re-read the explanation between questions to clarify, do you mean when you feel these things do you notice them? Or how often you feel them and notice them?

- For the sensations where I indicated that I pay very little attention to them, should I believe I have a reason to need to monitor this sensation then I will focus momentarily to determine whether or not it is genuinely needed or if I can wait to relieve it.

- I do often think about whether my heart is beating fast but it doesn't take up a lot of my time (frequent but not extended periods)

- my attention is very much bound by the activity in which I am engaging. Bodily discomforts rarely capture my attention.

- No, I try to be busy most of the time, therefore my attention is on other things. I try to ignore pain and bodily symptoms which would worry me.

- Realise when different things are going on in your life you pay greater or less attention to these signs.

- Some of these things occupy my mind 'a lot' of the time but not 'most' of the time. So when this has occurred I have usually put 'neither agree nor disagree' 
- The worse my anxiety is the more likely I am to think about the need to go to the toilet

- They shouldn't use the phrase 'most of the time' as most of the time your attention is focused on the activity you are doing. I answered the survey honestly but there is next to no usable data. 'Most of the time' should be put into certain contexts as in two of the questions above for example, "most of the time when you are eating". Perhaps asking how much time of the day you spend considering your feelings should open the questionnaire and then based on that proportion of time, how much is spent considering how hungry you are, how thirsty you are, etc.

- Thinking about coughing for me at the moment is just due to it being a coronavirus symptom

- This set of questions is confusing. Obviously no-one spends the majority of their time focusing on their body sensations. I think you should have phrased the question differently, e.g. when I am thirsty, I notice it never, rarely, often, always.

- Your example about needing to go to the toilet doesn't fit well with the question asked. The example and explanation focuses on how much attention one pays to the sensations yet most of the questions ask whether a sensation occupies most of your time. How much attention one pays to a sensation and how much of one's time a sensation occupies are two different questions.

\section{IAS}

- $\quad$ "I can always accurately perceive when I am sexually aroused.": I am physically unable to be aroused. How should I answer?

- Again a lot of the questions don't make sense. Feelings like hunger and thirst are perceptions so the question is asking whether we perceive a perception. Other 
questions you would only know how accurate your perception is by constantly monitoring your heart beat. For example, I believe that my heartbeat is fast but I don't know how accurate that is because I'm not monitoring it. Other questions ask about perception but they're not perceptions, for example it's either I'm breathing fast or I'm not, that's an observation. Again, I don't know how much usable data can be gained from this quiz.

- I didn't know how to answer the question about accurately perceiving pain - to my mind pain is a perception, not a bodily event and so don't know what the accuracy would be measured against.

- I find an inherent difficulty with some of the questions i.e. it may be that your heart never beats fast rather than an inability to "perceive it': the same with taste.

- It seems very insensitive to variations if I can only agree or disagree with an "always " statement . Some of these things I'm better at than others but almost none of them I can do always

- Sometimes I try to ignore body cues (e.g. when I am tired etc.) and so it is hard to differentiate when I am slightly tired. Would it be useful to have this factored in perhaps? Like how often one ignores body cues/does not believe them/how useful we find them and what impact this has on our ability to identify and respond to them?

- This questionnaire was a bit too absolute both in the statements given and the statements I had to choose from. This made it difficult to answer because the sensations and my perception of them can vary depending on intensity and my mood.

- Again, poor lexis choice. I can indicate how often I am aware of different feelings but you cannot measure my unawareness, which is equally important. To know how aware I am of bodily sensations I need to be monitored so my awareness can be 
measured. Perhaps I think I am very aware of how much I tap my foot but in fact I tap my foot a lot subconsciously so my answer above is inaccurate. Again, not sure how much usable data there is here.

- Having a chronic pain condition means I am always 'aware' of these things because they consume my life, however I am adept at pushing them to the back of my mind in order to continue to live a relatively normal life despite them.

- I felt this was a more accurate way of describing the attention I give these sensations than the first questionnaire.

- I thought this is if the need to urinate existed for example, how well would I perceive it?

- I was confused about what was meant by aware. I moved between the 3 interpretations suggested above but decided then that on the whole it must mean my accuracy at perceiving these sensations when they occur.

- I would expand your explanation of awareness at the top. Even though I know you wanted us to give answers in line with the third option, we weren't really led in that direction.

- It's a little hard for us to judge how HARD our hearts are beating...

- Not clear whether the term 'focus' refers to concentration or vision

- Occasionally and sometimes mean the same thing, different words should have been selected.

- Probably good to separate out frequency and how aware you are 'when it happens' because some of these things don't really happen to me so I don't think I'm aware of it but how would I know if it doesn't happen.

- The use of the word 'aware' was very confusing 
- To be honest I'm not sure exactly what you mean by "situation" - is it something happening as in "so and so happened and it caused a situation" or just normal things that happen during the day like lying in bed (and hearing digestion noise)?

- Very confusing

\section{[S13] Study 2: Examination of non-linear associations}

Non-linear associations were again investigated with accuracy as the dependent variable and attention as the independent variable. Where non-linear associations were examined in subsamples where the BPQ was interpreted as assessing accuracy, the BPQ was used as the independent variable for consistency. In the total sample, for the IAS and BPQ the inclusion of both quadratic $(p<.001)$ and cubic $(p=.049)$ trends significantly improved the model. For the IAS to IATS, only the inclusion of the quadratic term significantly improved the model fit $(p<.001)$. For those interpreting the BPQ as assessing attention $(\mathrm{N}=123)$, the inclusion of the quadratic and cubic trends did not significantly improve the model fit (all $p>.10$ ). For the IAS to IATS in this sample, only the inclusion of the quadratic term improved the model fit $(p<.001)$. For those interpreting the BPQ as assessing accuracy, only the inclusion of the quadratic term significantly improved the model fit for the relationship between the BPQ and IAS ( $p=.013)$. For the IAS to IATS, there was only a trend for the quadratic term to improve model fit $(p=.078)$ in this sample. In those who interpreted the BPQ as assessing how often the signal occurs, only the quadratic trend significantly improved the model for the relationship between the BPQ and IAS ( $p=.014)$. Likewise, only the quadratic trend significantly improved model fit for the IATS and IAS model $(p=.003)$. As in Study 1, where quadratic trends were observed these indicated that both high and low levels of self-reported interoceptive attention were associated with higher self-reported accuracy. Cubic trends remained difficult to interpret. 